Universidade de São Paulo

Instituto DE FísicA

\title{
Estudo dos Estágios Iniciais do Crescimento de Ge Sobre $\mathrm{Si}(100)$
}

Gustavo Martini Dalpian

Apresentada junto ao Instituto de Física da Universidade de São Paulo para a obtenção do título de Mestre em Ciências.

Banca Examinadora:

Prof. Dr. Adalberto Fazzio (Orientador)

Prof. Dr. Alain Andre Quivy - USP

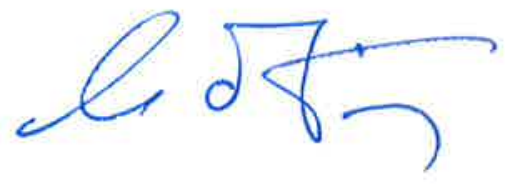
Prof. Dr. Alex Antonelli - UNICAMP

SÃO PAULO 2000

SBI-IFUSP

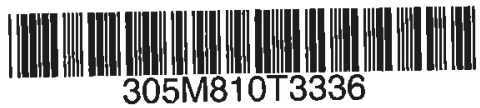

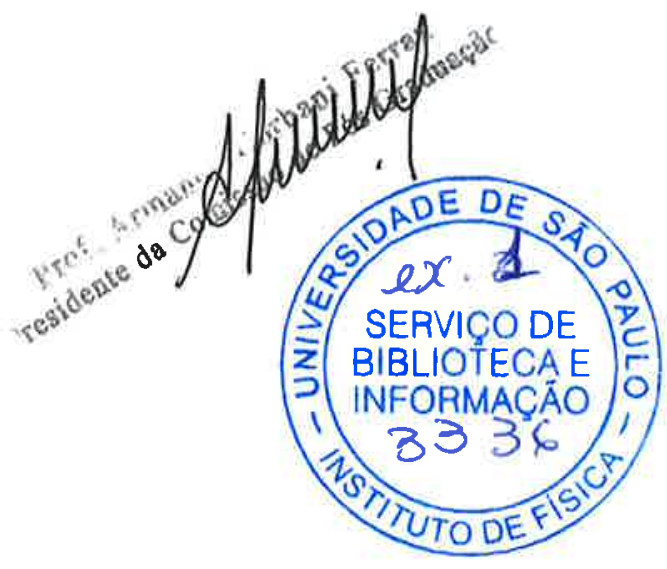


537.622

1) 149 e

$M$

$e x \cdot 1$

FICHA CATALOGRÁFICA

Preparada pelo Serviço de Biblioteca e Informação do Instituto de Física da Universidade de São Paulo

Dalpian, Gustavo Martini

Estudo dos Estágios Iniciais do Crescimento de Ge Sobre Si(100).

São Paulo, 2000.

Dissertação (Mestrado) - Universidade de São Paulo.

Instituto de Física - Departamento de Física dos Materiais

e Mecânica

Orientador: Prof. Dr. Adalberto Fazzio

Área de Concentração: Física do Estado Sólido

Unitermos: 1. Ge sobre Si(100); 2. Crescimento de

Semicondutores; 3. Método de Primeiros Princípios.

USP/IF/SBI-011/2000 
À Patricia e

Laura Cristina. 


\section{Conteúdo}

Agradecimentos iv

Resumo v v

Abstract vi

1 Introdução 1

2 Método de Cálculo de Energia Total 4

2.1 A Teoria do Funcional da Densidade(TFD) . . . . . . . . . 5

2.2 Pseudopotenciais de Norma Conservada . . . . . . . . . . . 12

2.2.1 Separação dos Pseudopotenciais . . . . . . . . . . 16

2.2.2 Transferibilidade . . . . . . . . . . . . . . 18

2.3 A Energia Total no Espaço dos Momentos . . . . . . . . . . 19

3 A Superfície $\operatorname{Si(100)} \quad 27$

3.1 Preparação da Superfície . . . . . . . . . . . . . . . . . . 28

3.2 Relaxação e Reconstrução . . . . . . . . . . . . . . . . . 29

4 Crescimento de Ge Sobre $\operatorname{Si(100)} \quad 35$

4.1 Revisão Bibliográfica . . . . . . . . . . . . . 36

4.2 Resultados . . . . . . . . . . . . . . . . 43

4.2 .1 Monômeros . . . . . . . . . . . . . . . 44

4.2 .2 Dímeros . . . . . . . . . . . . . . 5 50 
4.2 .3 Trímeros . . . . . . . . . . . . . . 55

5 Conclusão $\quad 61$

$\begin{array}{ll}\text { Bibliografia } & 63\end{array}$ 


\section{Agradecimentos}

Agradeço a todas as pessoas que me ajudaram durante o período relativo a este trabalho, em especial ao Anderson, Gilberto e Brito pela ajuda científica, computacional e também pela amizade. Ao Evandro, Evaldo e Ivan, que tornaram a minha convivência em São Paulo mais amena, através das conversas, discussões, pizzas e cervejas. Aos meus pais, que sempre me apoiaram muito em todos os estágios da minha formação.

Gostaria de agradecer especialmente ao Prof. Adalberto Fazzio e ao Prof. Antônio José Róque da Silva, por todas as oportunidades que me deram, por todas as informações que me passaram e pela preciosa orientação. Gostaria também demostrar meu respeito e admiração por estes professores, pela sua competência e seriedade no trabalho.

Agradeço também à FAPESP pelo suporte financeiro e ao CENAPAD-SP pelo suporte computacional. 


\section{Resumo}

A superfície '(100) do Silício é estudada através de métodos de primeiros princípios, baseados na Teoria do Funcional da Densidade e no Método dos Pseudopotenciais. Estudamos as propriedades eletrônicas e estruturais das principais reconstruções desta superfície. Obtivemos os principais sítios ligantes para pequenas estruturas de Germânio adsorvidas sobre ela. Para um átomo adsorvido o sítio mais estável encontrado foi o sítio $\mathbf{M}$, semelhante a resultados existentes na literatura. A diferença está na configuração dos dímeros de Si da superfície. Observamos que diferentes configurações destes dímeros nos forneciam diferentes sítios metaestáveis. O deslocamento dos dímeros da superfície é tratado então como um novo grau de liberdade para a adsorção de átomos, além da posição destes sobre a superfície. Também foi estudada a adsorção de alguns trímeros de Ge sobre a superfície, sendo que a estrutura 'encontrada como sendo a mais estável concorda muito bem com outros resultados teóricos e experimentais. 


\section{Abstract}

The (100) surface of Silicon is studied using First Principles methods, based on the Density Functional Theory and on the Pseudopotential method. We obtained the electronic and structural properties of the most important reconstructions of this surface and we also obtained the main bonding sites for the adsorption of small Ge structures on the surface. Site $\mathbf{M}$ was found as being the mọst stable for the monomers adsorption, similar to previous results on the literature. The main difference is on the buckling of the silicon surface dimers. We observed that different configurations of these buckling gave us different metastable adsorption sites. We then insert a new degree of freedom for this kind of adsorption, related to the buckling of the silicon surface dimers. We also studied the adsorption of Ge trimers on the surface, and the structure found as being the most stable agree very well with previous theoretical and experimetal results. 


\section{Capítulo 1}

\section{Introdução}

O nível ao qual a ciência e a tecnologia chegaram atualmente impressiona pela velocidade com a qual estas evoluíram. O mais interessante é que elas sempre andaram juntas, uma auxiliando a outra a ultrapassar barreiras.

A Física dos Materiais, em particular, foi responsável por algumas das mais importantes descobertas deste século. Podemos destacar aqui o transistor, responsável por uma imensa evolução na eletrônica; ligas ultraleves e ultraresistentes, usadas na indústria aeronáutica; biomateriais, utilizados na medicina; materiais magnéticos capazes de armazenar dados e lasers semicondutores, utilizados para os mais variados fins.

Todos estes exemplos, intensivamente aplicados nos dias de hoje, passaram primeiramente por exaustivos estudos básicos, que levaram a um completo entendimento dos problemas e que levaram a aplicações sem as quais hoje o homem não viveria.

O transistor, por exemplo, foi desenvolvido primeiramente na década de 40, através de estudos teóricos. O primeiro transistor, construído em 1947 tinha cerca de $1 \mathrm{~cm}$ de comprimento. Nos dias atuais, existem Circuitos Integrados deste tamanho que possuem da ordem de milhões de transistores no seu interior. $\mathrm{E}$ isto tudo é resultado da pesquisa básica na área de semicondutores. 
Este tipo de aplicações fez com que o estudo de materiais se desenvolvesse muito nos últimos tempos. Junto a isto, foram sendo desenvolvidos métodos cada vez mais precisos para se tratar estas estruturas numa escala microscópica. Outro fato que também impulsionou muito o estudo de materiais foi o desenvolvimento de computadores super potentes, capazes de fazer milhões de cálculos por segundo.

Pela maneira como o mundo está evoluindo, a tendência é que se desenvolvam equipamentos cada vez monores e que sejam cada vez mais potentes e mais eficazes. O estudo de novas ligas semicondutoras mostra que dispositivos de Arseneto de Gálio (GaAs) ou Silício-Germânio (SiGe) seriam muito mais velozes e muito mais eficazes do que os dispositivos atuais, de Silício.

O desenvolvimento de técnicas como o MBE (Molecular Beam Epitaxy), na qual diferentes átomos são jogados sobre uma superfície a uma taxa determinada, permitem a formação de ligas que não existem na natureza, e que possuem caracterísiticas interessantes tecnologicamente. Junto à esta técnica, foi desenvolvido na década passada uma técnica chamada Microscopia de Tunelamento Eletrônico (STM), que é capaz de observar estruturas ao nível atômico. Estas duas técnicas juntas nos permitem estudar a forma como estas ligas começam a se formar, o que permite também uma manipulação da estrutura do crescimento para se obterem características específicas.

É justamente este o objetivo principal deste trabalho. Estudamos os primeiros estágios do crescimento de Germânio sobre uma superfície de Silício, e obtivemos as posições preferenciais para a ligação de monômeros, dímeros e trímeros.

Para isto, utilizamos métodos de primeiros princípios, ou seja, métodos totalmente baseados na teoria quântica, livre de parâmetros empíricos. Este tipo de método nos fornece de forma bastante precisa as propriedades eletrônicas e estruturais do material. Para obter estes resultados, se faz necessário o uso de computadores, visto que para se obterem as informações sobre cada estrutura são necessários milhões de cálculos. 
No segundo capítulo deste trabalho, daremos uma noção dos métodos utilizados para fazer os cálculos. Explicaremos a Teoria do Funcional da Densidade, o Método dos Pseudopotenciais, o método utilizado para obtenção das energias e a forma utilizada para a dinâmica de núcleos e orbitais. No capítulo três, daremos uma noção das principais características estruturais e eletrônicas da superfície (100) do Silício, que é a superfície que utilizaremos para o crescimento. No capítulo quatro apresentaremos nossos resultados para adsorção de pequenas estruturas de Ge sobre $\mathrm{Si}(100)$. Paralelamente discutiremos alguns dos resultados mais relevantes de $\mathrm{Si}$ e Ge sobre $\mathrm{Si}(100)$ já existentes na literatura. 


\section{Capítulo 2}

\section{Método de Cálculo de Energia Total}

Todos os resultados apresentados aqui foram obtidos através de cálculos de primeiros princípios. Esse tipo de cálculo é o que segue mais rigorosamente a Mecânica Quântica, e por isso eles são chamados de "O Estado da Arte" no cálculo de estrutura eletrônica. Se analisarmos bem, a única informação preliminar que necessitamos é o número atômico dos átomos que compõe o sistema de interesse. Esta é a grande diferença entre cálculos de primeiros princípios e outros tipos de cálculos, como os semi-empíricos ou semiclássicos. Estes outros métodos necessitam de informações preliminares sobre as propriedades físicas do material, para que o cálculo seja ajustado de modo a fornecer resultados coerentes.

Apesar da Ḿecânica Quântica nos dar o caminho para a solução dos nossos problemas, é impossível chegarmos a uma solução exata, visto que sempre esbarraremos nos "problemas de muitos corpos". Estes problemas se originam do fato de a Hamiltoniana total conter um termo de interação entre elétrons, termo este que não é solúvel exatamente. Para transpor estes problemas, fazem-se necessárias algumas aproximações e alguns truncamentos, que serão descritos adiante. 
Nosso objetivo é calcular a energia total de um sistema em várias configurações, para que seja possível uma comparação e para que possamos concluir qual a configuração mais estável, ou, a energeticamente mais favorável. Para realizar tais cálculos, utilizamos a Teoria do Funcional da Densidade e o Método dos Pseudopotenciais. Os cálculos foram realizados utilizando-se o conjunto de programas desenvolvido pelo Fritz-Haber-Institut, na Alemanha [FHI96MD] [1].

Passaremos agora a descrever os métodos que foram utilizados e que são implementados neste programa.

\subsection{A Teoria do Funcional da Densidade(TFD)}

Hohenberg e Kohn (HK)[2] propuseram uma teoria para calcular a energia total de um conjunto de elétrons sob a influência de um potencial externo.

O primeiro passo é descrever a Hamiltoniana do sistema em questão:

$$
\hat{H}=\hat{T}+\hat{V}+\hat{U}
$$

onde

$$
\hat{T}=\frac{1}{2} \int \nabla \hat{\psi}^{\dagger}(\mathbf{r}) \nabla \hat{\psi}(\mathbf{r}) d \mathbf{r}=\frac{1}{2} \int \hat{\psi}^{\dagger}(\mathbf{r}) \nabla^{2} \hat{\psi}(\mathbf{r}) d \mathbf{r},
$$

é a energia cinética,

$$
\hat{V}=\int v(\mathbf{r}) \hat{\psi}^{\dagger}(\mathbf{r}) \hat{\psi}(\mathbf{r}) d \mathbf{r}
$$

é o potencial externo e

$$
\hat{U}=\frac{1}{2} \int \frac{1}{\left|\mathbf{r}-\mathbf{r}^{\prime}\right|} \hat{\psi}^{\dagger}(\mathbf{r}) \hat{\psi}^{\dagger}\left(\mathbf{r}^{\prime}\right) \hat{\psi}\left(\mathbf{r}^{\prime}\right) \hat{\psi}(\mathbf{r}) d \mathbf{r} d \mathbf{r}^{\prime}
$$


é o potencial coulombiano.

Note que aqui $\hat{\psi}(\mathbf{r})$ é o operador de campo, $\hat{n}(\mathbf{r})=\hat{\psi}^{\dagger}(\mathbf{r}) \hat{\psi}(\mathbf{r})$ é o operador densidade e $n(\mathbf{r})=\langle\phi|\hat{n}(\mathbf{r})| \phi\rangle$ é o valor esperado da densidade no estado fundamental.

A TFD se baseia em dois teoremas propostos por HK, que podem ser provados de uma maneira simples e elegante.

O primeiro teorema diz que o potencial externo $v(\mathbf{r})$ sentido pelos elétrons é um funcional único da densidade eletrônica $n(\mathbf{r})$.

Suponhamos que $|\phi\rangle$ seja o estado fundamental do sistema, com uma Hamiltoniana $\hat{H}$ referente a um potencial externo $v(\mathbf{r})$. Suponhamos também que exista um outro potencial externo $v^{\prime}(\mathbf{r})$, que resulta numa Hamiltoniana $\hat{H}^{\prime}$ e num estado $\left|\phi^{\prime}\right\rangle$. Os dois potenciais levam à mesma densidade $n(\mathbf{r})$. Note que devem ser considerados estados não degenerados.

Desta forma temos que:

$$
\langle\phi|\hat{H}| \phi\rangle\left\langle\left\langle\phi^{\prime}|\hat{H}| \phi^{\prime}\right\rangle=\left\langle\phi^{\prime}\left|\hat{H}^{\prime}\right| \phi^{\prime}\right\rangle+\left\langle\phi^{\prime}\left|\hat{V}-\hat{V}^{\prime}\right| \phi^{\prime}\right\rangle\right.
$$

ou

$$
E<E^{\prime}+\int\left[v(\mathbf{r})-v^{\prime}(\mathbf{r})\right] n(\mathbf{r}) d \mathbf{r} .
$$

Da mesma förma, se partirmos de $\left\langle\phi^{\prime}\left|\hat{H}^{\prime}\right| \phi^{\prime}\right\rangle$, teremos:

$$
E^{\prime}<E+\int\left[v^{\prime}(\mathbf{r})-v(\mathbf{r})\right] n(\mathbf{r}) d r .
$$

Somando (2.6) e (2.7), obteremos:

$$
E+E^{\prime}<E^{\prime}+E .
$$


Como pode ser visto, esta é uma relação impossível. Portanto, a suposição de que os potenciais $v(\mathbf{r})$ e $v^{\prime}(\mathbf{r})$ são diferentes é inconsistente. Assim sendo, o potencial externo $v(\mathbf{r})$ é um funcional único da densidade $n(\mathbf{r})$, visto que ela era a única quantidade igual nos dois casos descritos acima. Como somente exite um estado $|\phi\rangle$ e uma Hamiltoniana $\hat{H}$ para um dado $v(\mathbf{r})$, estes também são funcionais únicos da densidade eletrônica $n(\mathbf{r})$. Por causa deste fato, esta teoria se chama Teoria do Funcional da Densidade.

O segundo teorema proposto por $\mathrm{HK}$ diz que a energia do estado fundamental $E_{0}[n]$ é mínima para a densidade $n(\mathbf{r})$ correta.

Este teorema também é facilmente comprovado. Temos que:

$$
E_{0}[n]=\int v(\mathbf{r}) n(\mathbf{r}) d \mathbf{r}+F[n]
$$

onde

$$
F[n]=\langle\phi|\hat{T}+\hat{U}| \phi\rangle
$$

A energia para uma outra densidade $n^{\prime}(\mathbf{r})$ será:

$$
E\left[n^{\prime}\right]=\left\langle\phi^{\prime}|\hat{V}| \phi^{\prime}\right\rangle+\left\langle\phi^{\prime}|\hat{T}+\hat{U}| \phi^{\prime}\right\rangle
$$

Estamos supondo que o número total de partículas seja mantido constante, ou seja $N[n]=\int n(\mathbf{r}) d \mathbf{r}=N$ é constante. Por causa disso é que em (2.11) estamos usando $\hat{V}$ ao invés de $\hat{V}^{\prime}$.

Se reescrevermos (2.9) de forma que:

$$
E_{0}[n]=\langle\phi|\hat{V}| \phi\rangle+\langle\phi|\hat{T}+\hat{U}| \phi\rangle
$$

e juntarmos esta equação com (2.11), teremos que 


$$
E_{0}[n]<E\left[n^{\prime}\right]
$$

em virtude do valor esperado da energia ser menor para o estado $|\phi\rangle$, que é o autoestado correspondente à Hamiltoniana $\hat{H}$.

Portanto, a energia do estado fundamental será mínima para a densidade do sistema $n(\mathbf{r})$ correta.

Agora que possuímos estes dois teoremas em mãos, precisamos encontrar uma forma de descrever a Hamiltoniana em função de $n$. Começaremos pela função $F[n]$, que pode ser escrita como:

$$
F[n]=\langle\phi|\hat{T}+\hat{U}| \phi\rangle,
$$

sendo que

$$
\begin{aligned}
& \langle\phi|\hat{T}| \phi\rangle=T_{0}[n]+T_{x c}[n], \\
& \langle\phi|\hat{U}| \phi\rangle=U_{c}[n]+U_{x c}[n],
\end{aligned}
$$

onde $T_{o}[n]$ é a energia cinética de um sistema de partículas não interagentes, $U_{c}[n]$ é a energia coulombiana (termo de Hartree) e $E_{x c}[n]=$ $T_{x c}[n]+U_{x c}[n]$ é a energia de troca e correlação. Note que neste último termo $\left(E_{x c}[n]\right)$, também existe uma correção para a energia cinética.

O termo de troca e correlação não possui uma fórmula analítica exata. Para contornar esse problema, fazemos uma aproximação supondo que a densidade $n(\mathbf{r})$ varia muito lentamente, de forma que a energia de troca e correlação somente dependerá da densidade eletrônica local. Desta forma

$$
E_{x c}[n]=\int n(\mathbf{r}) \varepsilon_{x c}(n(\mathbf{r})) d \mathbf{r} .
$$


Esta aproximação é chamada de Aproximação da Densidade Local. $\varepsilon_{x c}$ é a energia de troca e correlação por elétron, e ela é calculada como se fosse a energia de exchange e correlação de um gás de elétrons de densidade uniforme $n$, igual à densidade $n(\mathbf{r})$ em questão. Esta energia deve ser calculada utilizando-se algum método alternativo.

Um outro problema que ainda não abordamos é que somente o mínimo valor da energia possui um significado físico na TFD, e ainda não foi proposto nenhum método para se determinar este mínimo. Para solucionar este problema, Kohn e Sham[3] propuseram o seguinte método:

Sabe-se que para o valor mínimo da energia, a variação dela será zero. Então:

$$
\delta E_{0}(n)=0
$$

Utilizando o método dos multiplicadores de Lagrange e a condição de que $N=\int n(\mathbf{r}) d \mathbf{r}$ é uma constante, teremos

$$
\delta\left[E_{0}(n)-\varepsilon N\right]=0,
$$

onde $\varepsilon$ é um multiplicador de Lagrange.

Como $E_{0}(n)$ é

$$
E_{0}(n)=\int v(\mathbf{r}) n(\mathbf{r}) d \mathbf{r}+T_{0}[n]+\iint \frac{n(\mathbf{r}) n\left(\mathbf{r}^{\prime}\right)}{\left|\mathbf{r}-\mathbf{r}^{\prime}\right|} d \mathbf{r} d \mathbf{r}^{\prime}+E_{x c}(n)
$$

então

$\delta\left[\int v(\mathbf{r}) n(\mathbf{r}) d \mathbf{r}+T_{0}[n]+\iint \frac{n(\mathbf{r}) n\left(\mathbf{r}^{\prime}\right)}{\left|\mathbf{r}-\mathbf{r}^{\prime}\right|} d \mathbf{r} d \mathbf{r}^{\prime}+E_{x c}(n)-\varepsilon \int n(\mathbf{r}) d \mathbf{r}\right]=0$,

$\mathrm{ou}$ 


$$
\begin{gathered}
\int \delta n(\mathbf{r}) v(\mathbf{r}) d \mathbf{r}+\int \frac{\delta T_{0}[n]}{\delta n} \delta n d \mathbf{r}+2 \iint \frac{\delta n(\mathbf{r}) n\left(\mathbf{r}^{\prime}\right)}{\left|\mathbf{r}-\mathbf{r}^{\prime}\right|} d \mathbf{r} d \mathbf{r}^{\prime}+\int \frac{\delta E_{x c}(n)}{\delta n} \delta n d \mathbf{r}- \\
-\varepsilon \int \delta n(\mathbf{r}) d \mathbf{r}=0, \quad(2.22) \\
\int \delta n(\mathbf{r})\left[v(\mathbf{r})+\frac{\delta T_{0}[n]}{\delta n}+2 \int \frac{n\left(\mathbf{r}^{\prime}\right)}{\left|\mathbf{r}-\mathbf{r}^{\prime}\right|} d \mathbf{r}^{\prime}+\frac{\delta E_{x c}(n)}{\delta n}-\varepsilon\right] d \mathbf{r}=0,
\end{gathered}
$$

Definimos agora um conjunto de funções ortonormais $\varphi_{i}(\mathbf{r})$, de forma que a densidade $n(\mathbf{r})$ possa ser definida por

$$
n(\mathbf{r})=\sum_{i=1}^{N} \varphi_{i}^{*}(\mathbf{r}) \varphi_{i}(\mathbf{r})
$$

e então

$$
\delta n(\mathbf{r})=\delta \varphi_{i}^{*}(\mathbf{r}) \varphi_{i}(\mathbf{r})
$$

assim teremes

$$
\int \delta \varphi_{i}^{*}(\mathbf{r})\left[v(\mathbf{r})+\frac{\delta T_{0}[n]}{\delta n}+2 \int \frac{n\left(\mathbf{r}^{\prime}\right)}{\left|\mathbf{r}-\mathbf{r}^{\prime}\right|} d \mathbf{r}^{\prime}+\frac{\delta E_{x c}(n)}{\delta n}-\varepsilon\right] \varphi_{i}(\mathbf{r}) d \mathbf{r}=0
$$

A energia cinética será dada por

$$
T_{0}[n]=-\frac{1}{2} \sum_{i} \int d \mathbf{r} \varphi_{i}^{*}(\mathbf{r}) \nabla^{2} \varphi_{i}(\mathbf{r})
$$

Como $\delta \varphi_{i}^{*}(\mathbf{r})$ é arbitrário, obteremos as seguintes equações de Schrödinger de uma partícula: 


$$
\left[-\frac{1}{2} \nabla^{2}+v(\mathbf{r})+2 \int \frac{n\left(\mathbf{r}^{\prime}\right)}{\left|\mathbf{r}-\mathbf{r}^{\prime}\right|} d \mathbf{r}^{\prime}+\frac{\delta E_{x c}(n)}{\delta n}\right] \varphi_{i}(\mathbf{r})=\varepsilon_{i} \varphi_{i}(\mathbf{r}) .
$$

Essas equações são conhecidas como Equações de Kohn-Sham. É importante enfatizar que as autofunções $\varphi_{i}(\mathbf{r})$ não possuem nenhuma função além de determinar a densidade eletrônica do estado fundamental.

Para se obter o valor da energia no estado fundamental, multiplicamos a equação (2.28) por $\varphi_{i}^{*}(\mathbf{r})$ e integramos em $\mathbf{r}$ :

$$
\int \varphi_{i}^{*}(\mathbf{r})\left[-\frac{1}{2} \nabla^{2}+v(\mathbf{r})+2 \int \frac{n\left(\mathbf{r}^{\prime}\right)}{\left|\mathbf{r}-\mathbf{r}^{\prime}\right|} d \mathbf{r}^{\prime}+\frac{\delta E_{x c}(n)}{\delta n}\right] \varphi_{i}(\mathbf{r}) d \mathbf{r}=\int \varphi_{i}^{*}(\mathbf{r}) \varepsilon_{i} \varphi_{i}(\mathbf{r}) d \mathbf{r}
$$

Somando sobre todos os elétrons obtemos:

$$
\begin{aligned}
& \sum_{i} \int \varphi_{i}^{*}(\mathbf{r})\left(-\frac{1}{2} \nabla^{2}\right) \varphi_{i}(\mathbf{r}) d \mathbf{r}+\sum_{i} \int \varphi_{i}^{*}(\mathbf{r}) v(\mathbf{r}) \varphi_{i}(\mathbf{r}) d \mathbf{r}+ \\
& +2 \sum_{i} \iint \frac{n(\mathbf{r}) n\left(\mathbf{r}^{\prime}\right)}{\left|\mathbf{r}-\mathbf{r}^{\prime}\right|} d \mathbf{r} d \mathbf{r}^{\prime}+\sum_{i} \int \varphi_{i}^{*}(\mathbf{r}) \frac{\delta E_{x c}(n)}{\delta n} \varphi_{i}(\mathbf{r}) d \mathbf{r}=\sum_{i} \varepsilon_{i} .
\end{aligned}
$$

Substituindo a equação (2.20) na equação acima:

$$
E_{0}(n)+\iint \frac{n(\mathbf{r}) n\left(\mathbf{r}^{\prime}\right)}{\left|\mathbf{r}-\mathbf{r}^{\prime}\right|} d \mathbf{r} d \mathbf{r}^{\prime}-E_{x c}(n)+\sum_{i} \int \varphi_{i}^{*}(\mathbf{r}) \frac{\delta E_{x c}(n)}{\delta n} \varphi_{i}(\mathbf{r}) d \mathbf{r}=\sum_{i} \varepsilon_{i},
$$

fazendo

$$
\frac{\delta E_{x c}}{\delta n}=\frac{d\left(n \varepsilon_{x c}(n)\right)}{d n}=\mu_{x c},
$$

obtemos para o valor da energia 


$$
E_{0}(n)=\sum_{i=1}^{N} \varepsilon_{i}-\iint \frac{n(\mathbf{r}) n\left(\mathbf{r}^{\prime}\right)}{\left|\mathbf{r}-\mathbf{r}^{\prime}\right|} d \mathbf{r} d \mathbf{r}^{\prime}+\int n(\mathbf{r})\left[\varepsilon_{x c}(n(\mathbf{r}))-\mu_{x c}(n(\mathbf{r}))\right] d \mathbf{r}
$$

Como podemos ver, este é um típico caso de um problema de muitos corpos que é transformado em problemas mais simples, de uma única partícula. Desta forma, podemos calcular a densidade eletrônica do nosso sistema, e com isto, podemos também obter todas as outras propriedades.

Um outro fato que deve ser enfatizado é que as energias $\varepsilon_{i}$ não correspondem as energias independentes dos elétrons. Somente a sua soma possui um significado como parte da energia para o estado fundamental.

\subsection{Pseudopotenciais de Norma Conservada}

A interação dos elétrons com os núcleos atômicos é o assunto desta seção. Nos cálculos realizados por nós as funções de onda são descritas por expansões em ondas planaśs. Entretanto, seria muito caro descrevê-las totalmente, em vista da grande quantidade de nodos que estas possuem na região nuclear. O método dos pseudopotenciais faz uma simplificação que torna os cálculos mais viáveis computacionalmente, sem perder as características dos átomos que iremos trabalhar.

Este método se baseia no método OPW, onde os átomos são divididos em duas partes: (i) os elétrons de valênica, aqueles da última camada eletrônica (maior número quântico principal), que são responsáveis pelas ligações químicas; (ii) o caroço iônico, constituído pelo núcleo e pelos elétrons que não são de valência.

A Método dos Pseudopotenciais atua na região do caroço iônico, eliminando a necessidade de se introduzirem os estados do caroço. Desta forma, 
a função de onda nesta região será substituída por uma função suave e sem nodos, que pode ser descrita por um conjunto de ondas planas muito menor.

Apesar de não descrever corretamente os potenciais na região do caroço, as funções de onda são corretamente descritas na região de valência. Estes pseudopotenciais são contruídos de forma a seguirem as seguintes propriedades:

- os autovalores real e pseudo devem ser iguais;

- as autofunções real e pseudo devem ser iguais para posições acima de um raio de corte $r_{c}$;

- as integrais de 0 a $r$ das densidades de carga real e pseudo devem ser iguais para $r>r_{c}$ (conservação da norma);

- a derivada logarítmica das funções de onda real e pseudo e as suas primeiras derivadas com relação à energia devem ser iguais para $r>r_{c}$;

Este tipo de pseudopotenciais são chamados de Pseudopotenciais de Norma conservada, e foram descritos por Haman, Schluter e Chiang[4] (HSC) e posteriormente por Bachelet, Haman e Schluter[5] (BHS).

Para obtermos pseudopotenciais do tipo HSC, resolve-se primeiramente o átomo em questão, utilizando-se um método de primeiros princípios (Teoria do Funcional da Densidade). Com isto, obtemos o valor do potencial real $V(r)$ e também a função de onda:

$$
\Psi(\mathbf{r})=\left[\frac{u_{\ell}(r)}{r}\right] Y_{\ell m}\left(\Omega_{r}\right) .
$$

Como se trata de um problema com simetria esférica, a função de onda utilizada será somente a parte radial $u_{\ell}(r)$.

O pseudopotencial é construído da seguinte forma:

$$
V_{1 \ell}^{p s}(r)=\left[1-f\left(\frac{r}{r_{c \ell}}\right)\right] V(r)+c_{l} f\left(\frac{r}{r_{c \ell}}\right),
$$


onde $r_{c \ell}$ é o raio de corte e $f(x)$ é uma função de corte responsável por forçar $V_{1 \ell}^{p s}(r)$ tender para $V(r)$ para $r>r_{c \ell}$. O raio de corte sempre é escolhido de forma a estar entre o último nodo da função de onda real e o último pico.

Substituindo este potencial $V_{1 l}^{p s}$ na equação radial de Schrödinger com um autovalor $\varepsilon_{\ell}$, obteremos uma autofunção sem nodos $w_{1 \ell}$. A constante $c_{\ell}$ serve para que o pseudo-autovalor $\varepsilon_{1 \ell}$ seja igual ao autovalor real $\varepsilon_{\ell}$.

Para que as funções de onda real e pseudo sejam iguais acima do raio de corte, introduzimos uma constante de forma que

$$
\gamma_{\ell} w_{1 \ell}(r)=u_{\ell}(r)
$$

Para satisfazer às outras propriedades, mudamos a pseudo-função de onda de forma que

$$
w_{2 \ell}(r)=\gamma_{\ell}\left[w_{1 \ell}(r)+\delta_{\ell} g_{\ell}\left(\frac{r}{r_{c \ell}}\right)\right],
$$

onde $g_{\ell}$ é uma função de corte responsável por tornar o pseudopotencial finito na origam e $\delta_{\ell}$ é uma constante que garante a normalização, ou seja:

$$
\gamma_{\ell}^{2} \int_{0}^{\infty}\left[w_{1 \ell}(r)+\delta_{\ell} g_{\ell}\left(\frac{r}{r_{c \ell}}\right)\right]^{2} d r=1 .
$$

O pseudopotencial final $V_{2 \ell}^{p s}(r)$ é encontrado invertendo-se a equação radial de Schrödinger, com função de onda $w_{2 \ell}(r)$ e autovalor $\varepsilon_{1 \ell}$. As funções de onda real e pseudo podem ser vistas na Figura (2.1), com seus respectivos raios de corte, para o Silício. Os pseudopotenciais podem ser vistos na Figura 2.2.

As funções de corte usadas possuem a seguinte forma: 


$$
\begin{gathered}
f(x)=\exp \left(-x^{3.5}\right), \\
g_{\ell}(x)=x^{\ell+1} \exp \left(-x^{4}\right) .
\end{gathered}
$$

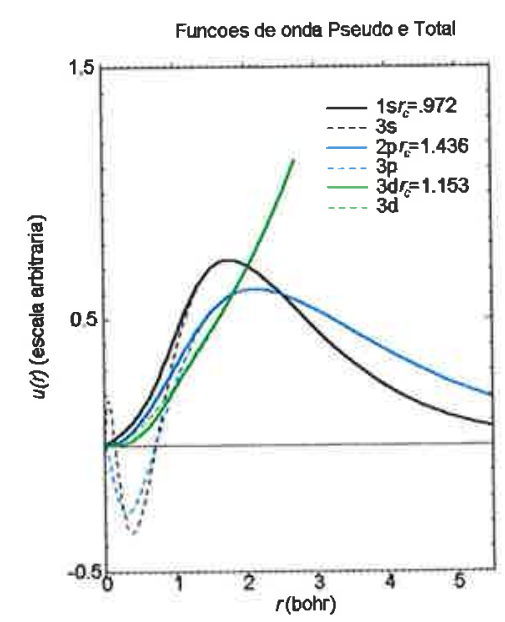

Figura 2.1: Funções de onda real (linha pontilhada) e pseudo (linha sólida). Os raios de corte utilizados podem ser vistos no detalhe.

Existem ainda outros tipos de pseudopotenciais, que são construídos de maneira diferente, mas que seguem a mesma filosofia dos descritos por HSC. Podemos citar o trabalho de Troullier e Martins [6], que introduziram alguns vínculos aos pseudopotenciais, como o fato da curvatura do pseudopotencial ser zero na origem e também das quatro primeiras derivadas das funções de onda real e pseudo serem iguais no raio de corte. Como os pseudopotenciais 


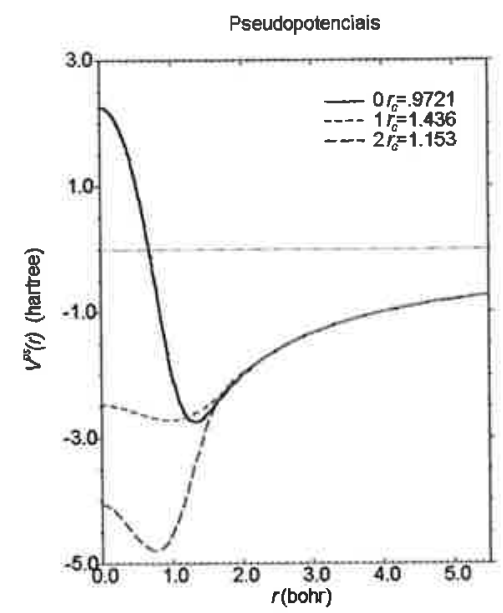

Figura 2.2: Pseudopotenciais para as diferentes componentes do momento angular $\ell=1,2,3$.

usados neste trabalho foram todos gerados utilizando o método de BHS, não entraremos em maiores detalhes sobre os pseudopotenciais de Troullier e Martins.

\subsubsection{Separação dos Pseudopotenciais}

Se observarmos a Figura 2.2, notaremos que todas as componentes $V_{\ell}^{p s}(r)$ tendem para o mesmo valor (o do potencial Coulombiano $\left(\frac{-Z}{r}\right)$ ) para grandes valores de $r$. Isto nos permite então descrever o pseudopotencial como um termo local, independente de $\ell$ e de longo alcance, somado de termos semilocais, de curto alcance. $\mathrm{O}$ termo local normalmente é descrito pelo termo com o maior momento angular $\ell$. Teremos então que: 


$$
V_{\ell}^{p s}(r)=V^{l o c}(r)+\delta V_{\ell}^{s l}(r)
$$

onde

$$
V^{l o c}(r)=V_{\ell=l o c}^{p s}(r) \quad \text { e } \quad \delta V_{\ell}^{s l}(r)=V_{\ell}^{p s}(r)-V_{\ell_{l o c}}^{p s}(r) .
$$

Onde $\delta V_{\ell}^{s l}(r)$ representa os termos semi-locais.

Considerando isto, Kleinman e Bylander [7] propuseram uma separação totalmente não local. Eles observaram que tornando a forma do pseudopotencial mais complexa no espaço real, resultava numa grande diminuição do número de integrais no espaço recíproco.

O termo não local é utilizado no lugar do termo semi-local $\delta V_{\ell}^{s l}(r)$. Este termo é definido da seguinte forma:

$$
\delta V_{n l}\left(\mathbf{r}, \mathbf{r}^{\prime}\right)=\sum_{\ell, m} \delta V_{s l, \ell}^{i o n}(r) R_{\ell}^{p s}(r) Y_{\ell m}(\mathbf{r})\left\langle\delta V_{s l, \ell}\right\rangle^{-1} Y_{\ell m}^{*}\left(\mathbf{r}^{\prime}\right) R_{\ell}^{p s}\left(r^{\prime}\right) \delta V_{s l, \ell}^{i o n}\left(r^{\prime}\right)
$$

onde a constante de normalização é dada por

$$
\left\langle\delta V_{s l, \ell}\right\rangle=\int d \mathbf{r} R_{\ell}^{p s}(r) Y_{\ell m}^{*}(\mathbf{r}) \delta V_{s l, \ell}^{i o n}(r) Y_{\ell m}(\mathbf{r}) R_{\ell}^{p s}(r) .
$$

Esta mudança não altera a forma original do pseudopotencial, visto que

$$
\int d \mathbf{r} \delta V_{n l}\left(\mathbf{r}, \mathbf{r}^{\prime}\right) R_{\ell}^{p s}(r) Y_{\ell m}(\mathbf{r})=\delta V_{s l, \ell}^{i o n}(r) R_{\ell}^{p s}(r) Y_{\ell m}(\mathbf{r})
$$

Como já foi dito antes, esta aparente "complicação" do problema na realidade faz com que ele fique mais fácil de ser resolvido. Utilizando o termo semi-local, com uma base $N$-dimensional, são necessárias $\frac{\left(N^{2}+N\right)}{2}$ integrais, enquanto que com o termo não local, são necessárias $N$ integrais. 


\subsubsection{Transferibilidade}

Uma vez que o pseudopotencial é gerado, por construção ele já reproduz corretamente os estados de valência do átomo livre. Entretanto, isto não acarreta necessariamente numa descrição eficaz do potencial do átomo em diferentes estruturas, como moléculas ou sólidos.

Esta propriedade dos pseudopotenciais poderem ser tranferidos para outras estruturas depende de muitos fatores, entre eles o valor do raio de corte escolhido e a forma como este pseudopotencial é construído. Deve-se observar que quanto menor for o raio de corte, mais eficaz será a descrição do pseudopotencial.

Quando os pseudopotenciais usados neste trabalho foram gerados, foram feitos vários testes, a firm de verificar a transferibilidade destes para sólidos e moléculas. Para sólidos foram feitos cálculos a fim de se obterem algumas propriedades físicas bem determinadas experimentalmente para o Silício e o Germânio, como a distância de ligação entre átomos no sólido e o bulk modulus destes.

Para o Silício, obtivemos que a distância de ligação entre os átomos seria $2.33 \AA$, ou $-1 \%$ em relação ao resultado experimental. Para o bulk modulus foi encontrada a mesma diferença percentual. No caso do Germânio, encontramos que a distância de ligação mais estável seria $2.42 \AA$, ou $-1,3 \%$ do valor experimental. Para o bulk modulus encontramos uma diferença de $+5 \%$ em relação ao resultado experimental.

Para moléculas, simulamos as moléculas de $\mathrm{Si}_{2} \mathrm{H}_{6}, \mathrm{Ge}_{2} \mathrm{H}_{6}$ e $\mathrm{SiGeH}_{6}$ e dos radicais $\mathrm{SiH}_{3}$ e $\mathrm{GeH}_{3}$. Os radicais foram simulados utilizando-se parte da estrutura relaxada das outras moléculas, sem que os átomos fossem relaxados novamente.

As distâncias atômicas e os ângulos entre os átomos concordaram perfeitamente com resultados da literatura.

Além disso, estes cálculos nos forneceram informações a respeito da ener- 
gia das ligações Si-Si, Ge-Ge e Ge-Si. Para isso, observamos as diferenças de energia entre as moléculas e os seus radicais constituintes separados, sem que eles fossem novamente relaxados. Obtivemos que a ligação entre átomos de Si vale cerca de $4.36 \mathrm{eV}$, entre os átomos de Ge vale $4.12 \mathrm{eV}$ e para a ligação Ge-Si foi encontrada uma energia de $4.25 \mathrm{eV}$.

\subsection{A Energia Total no Espaço dos Momentos}

Apesar de termos em mãos um método preciso para se obter a energia total de um sistema, o número de integrais que precisam ser resolvidas para se obter tal objetivo é muito grande. Para tentar tornar este problema mais simples, Ihm, Zunger e Cohen [8] propuseram, em 1979, um método mais simples para tratar o problema. A sugestão dos autores é que a energia total seja calculada no espaço recíproco, ou, no espaço dos momentos.

Para isto, torna-se necessário que façamos a transformada de Fourier de todos os termos da energia total para o espaço recíproco. A energia total de um sistema no espaço real é dada por:

$$
\begin{gathered}
E_{\text {total }}=T+V+\int E_{x c}(r) d^{3} r \\
V=\sum_{i, \mu, l} \int \psi_{i}(\mathbf{r}) U_{p s, \ell}\left(\mathbf{r}-\mathbf{R}_{\mu}\right) \hat{P}_{\ell} \psi_{i}(\mathbf{r}) d^{3} \mathbf{r}+\frac{1}{2} \iint \frac{2 n(\mathbf{r}) n\left(\mathbf{r}^{\prime}\right)}{\left|\mathbf{r}-\mathbf{r}^{\prime}\right|} d^{3} \mathbf{r} d^{3} \mathbf{r}^{\prime}+ \\
+\frac{1}{2} \sum_{\mu, \nu} \frac{2 Z^{2}}{\left|\mathbf{R}_{\mu}-\mathbf{R}_{\nu}\right|}
\end{gathered}
$$

onde $T$ é a energia cinética, dada por (2.27), $V$ é o potencial eletrostático, definido em (2.47) como a energia referente aos pseudopotenciais $U_{p s, \ell}$, onde $\ell$ se refere ao momento angular, somado da interação entre os elétrons mais 
a interação entre os núcleos. O último termo do lado direito de (2.46) é a energia de correlação e troca.

As funções de onda utilizadas serão descritas por expansões em ondas planas, de forma que

$$
\psi_{i}(\mathbf{r})=\sum_{G} C\left(\mathbf{k}_{i}+\mathbf{G}\right) \exp \left(i\left(\mathbf{k}_{i}+\mathbf{G}\right) \mathbf{r}\right)
$$

Assim, podemos definir o potencial coulombiano eletrônico e a densidade de carga no espaço recíproco como sendo

$$
\begin{aligned}
V_{\text {Coul }}(\mathbf{G}) & =\sum_{\mathbf{r}} V_{\text {Coul }}(\mathbf{r}) \exp (-i \mathbf{G r}) \\
n,(\mathbf{G}) & =\sum_{\mathbf{r}} n(\mathbf{r}) \exp (-i \mathbf{G r})
\end{aligned}
$$

Desta forma, através da Equação de Poisson, obtemos que

$$
V_{\text {coul }}(\mathbf{G})=\frac{8 \pi n(\mathbf{G})}{\mathbf{G}^{2}}
$$

Fazendo com que $\Omega$ seja o volume total do sistema, $\mathrm{N}$ o número de átomos e $\Omega_{a t}=\Omega / N$, podemos escrever os termos de (2.46) como:

$$
\begin{gathered}
\frac{1}{2} \iint \frac{2 n(\mathbf{r}) n\left(\mathbf{r}^{\prime}\right)}{\left|\mathbf{r}-\mathbf{r}^{\prime}\right|} d^{3} \mathbf{r} d^{3} \mathbf{r}^{\prime}=\frac{1}{2} \Omega \sum_{G} V_{\text {coul }}(\mathbf{G}) n(\mathbf{G}), \\
\mu_{x c}(\mathbf{r})=\sum_{G} \mu_{x c}(\mathbf{G}) \exp (i \mathbf{G r})
\end{gathered}
$$




$$
\sum_{i} \int \psi_{i}^{*}(\mathbf{r})\left(-\nabla^{2}\right) \psi_{i}(r) d^{3} \mathbf{r}=\Omega \sum_{i, \mathbf{G}}\left|C\left(\mathbf{k}_{i}+\mathbf{G}\right)\right|^{2}\left(\mathbf{k}_{i}+\mathbf{G}\right)^{2}
$$

O termo relativo aos pseudopotenciais, primeiro termo do lado direito da equação (2.47), será no espaço recíproco:

$\Omega\left(\sum_{\mathbf{G}} S(\mathbf{G}) U_{p s}(\mathbf{G}) n(\mathbf{G})+\sum_{i, \ell, \mathbf{G}_{,} \mathbf{G}^{\prime}} C^{*}\left(\mathbf{k}_{i}+\mathbf{G}\right) C\left(\mathbf{k}_{i}+\mathbf{G}\right) S\left(\mathbf{G}-\mathbf{G}^{\prime}\right) U_{p s, \ell, \mathbf{k}_{i}+\mathbf{G}, \mathbf{k}_{i}+\mathbf{G}^{\prime}}^{\prime}\right)$

onde

$$
S(\mathbf{G})=\sum_{\mu} \frac{\exp \left(i \mathbf{G R}_{\mu}\right)}{N}
$$

é o fator de estrutura, $U_{p s}(\mathbf{G})$ é a parte local, ou de longo alcance, do pseudopotencial e $U_{p s, \ell, \mathbf{k}_{i}+\mathbf{G}, \mathbf{k}_{i}+\mathbf{G}^{\prime}}^{\prime}$ é a parte não-local, ou de curto alcance, do pseudopotencial.

Juntando as equações (2.51), (2.52), (2.53) e (2.55) numa equação de uma partícula (2.28), obtemos:

$$
\sum_{\mathbf{G}^{\prime}}\left[\left(\mathbf{k}_{i}+\mathbf{G}^{\prime}\right)^{2} \delta_{\mathbf{G G}^{\prime}}+V_{\mathbf{G G}^{\prime}}^{i}\right] C\left(\mathbf{k}_{i}+\mathbf{G}^{\prime}\right)=\varepsilon_{i} C\left(\mathbf{k}_{i}+\mathbf{G}\right)
$$

onde

$$
\begin{aligned}
V_{\mathbf{G G}^{\prime}}^{i}=V_{\text {coul }}\left(\mathbf{G}-\mathbf{G}^{\prime}\right)+ & \mu_{x c}\left(\mathbf{G}-\mathbf{G}^{\prime}\right)+S\left(\mathbf{G}-\mathbf{G}^{\prime}\right) \times \\
& \times\left[U_{p s}\left(\mathbf{G}-\mathbf{G}^{\prime}\right)+\sum_{\ell} U_{p s, \ell, \mathbf{k}_{i}+\mathbf{G}, \mathbf{k}_{i}+\mathbf{G}^{\prime}}^{\prime}\right] .
\end{aligned}
$$


A energia total no espaço recíproco será dada então por ${ }^{1}$

$$
E_{t o t}=\sum_{i} \varepsilon_{i}-\Omega\left[\frac{1}{2} \sum_{\mathbf{G}} V_{c o u l}(\mathbf{G}) n(\mathbf{G})+\frac{1}{4} \sum_{\mathbf{G}} \mu_{x c}(\mathbf{G}) n(\mathbf{G})\right]+\frac{1}{2} \sum_{\mu, \nu} \frac{2 Z^{2}}{\left|\mathbf{R}_{\mu}-\mathbf{R}_{\nu}\right|}
$$

Entretanto, para esta fórmula poder ser implementada computacionalmente são necessárias algumas mudanças, em virtude de $V_{\text {coul }}(0), U_{p s}(0)$ e $\frac{1}{2} \sum_{\mu, \nu} \frac{2 Z^{2}}{\left|\mathbf{R}_{\mu}-\mathbf{R}_{\nu}\right|}$ serem quantidades individualmente divergentes. A fim de resolver este problema, resolvemos (2.57) com $V_{\text {coul }}(0)$ e $U_{p s}(0)$ iguais a zero. Isto corresponde a um deslocamento do referencial do potencial, mas isto é corrigido se, para pequenos $|\mathbf{G}|$, o potencial local for dado por

$$
U_{p s}(\mathbf{G})=-\frac{8 \pi Z}{\Omega_{a t} \mathbf{G}^{2}}+\alpha_{1}+\mathcal{O}\left(\mathbf{G}^{n}\right) \quad n \geq 3
$$

onde $\Omega_{a t}$ é o volume por átomo e

$$
\alpha_{1}=\lim _{G \rightarrow 0}\left[U_{p s}(\mathbf{G})+\frac{8 \pi Z}{\Omega_{a t} \mathbf{G}^{2}}\right]=\frac{1}{\Omega_{a t}} \int\left[U_{p s}(\mathbf{r})+\frac{2 Z}{\mathbf{r}}\right] d \mathbf{r} .
$$

Assim como a expansão para o pseudodotencial não local em (2.60), a densidade de carga também deve ser expandida, de forma que

$$
n(\mathbf{G})=\frac{Z}{\Omega_{a t}}+\beta \mathbf{G}^{2}+\mathcal{O}\left(\mathbf{G}^{n}\right) \quad n \geq 3
$$

onde

$$
\beta=\lim _{G \rightarrow 0} \frac{\Omega_{a t}}{8 \pi}\left(V_{\text {coul }}(\mathbf{G})-\frac{8 \pi Z}{\Omega_{a t} \mathbf{G}^{2}}\right)=\frac{\Omega_{a t}}{8 \pi} \frac{1}{\Omega} \iint \frac{2\left[n(\mathbf{r})-\frac{Z}{\Omega_{a t}}\right]}{\left|\mathbf{r}-\mathbf{r}^{\prime}\right|} d \mathbf{r} d \mathbf{r}^{\prime}
$$

\footnotetext{
${ }^{1}$ Consideramos aqui que $E_{x c}=\frac{3}{4} n(\mathbf{r}) \mu_{x c}(\mathbf{r})$.
} 
Este último termo introduz um alto índice de dificuldade para a solução das equações. Entretanto, $\beta$ não entra na fórmula final da energia, visto que tanto as interações entre os elétrons como a interação dos núcleos com os elétrons contêm $\beta$. Desta forma, os termos se cancelam.

A interação entre os íons é expressa supondo-se que o potencial eletrostático médio é zero, ou

$$
\frac{1}{2} \sum_{\nu} \frac{2 Z^{2}}{\left|\mathbf{R}_{\nu}\right|}=\gamma_{E w a l d}+\frac{1}{2} \lim _{\mathbf{G} \rightarrow 0} \frac{8 \pi Z^{2}}{\Omega_{a t} \mathbf{G}^{2}}
$$

onde

$$
\gamma_{\text {Ewald }}=\frac{1}{2} \sum_{\nu} \frac{2 Z^{2}}{\left|\mathbf{R}_{\nu}\right|}-\frac{1}{2} \lim _{\mathbf{G} \rightarrow 0} \frac{8 \pi Z^{2}}{\Omega_{a t} \mathbf{G}^{2}}=\frac{1}{2}\left(\sum_{\nu} \frac{2 Z^{2}}{\left|\mathbf{R}_{\nu}\right|}-\frac{1}{\Omega_{a t}} \int \frac{2 Z^{2}}{\mathbf{r}} d \mathbf{r}\right) .
$$

Nas equações (2.64) e (2.65), nos somatórios sobre $\nu$, devem ser excluidos os termos com $\mathbf{R}_{\nu}=0$.

Juntando-se as equações (2.60), (2.62) e (2.64), obtemos que a soma dos termos antes divergentes será:

$$
\lim _{\mathbf{G} \rightarrow 0} \Omega_{a t}\left[\frac{1}{2} V_{c o u l}(\mathbf{G}) n(\mathbf{G})+U_{p s}(\mathbf{G}) n(\mathbf{G})\right]+\frac{1}{2} \sum_{\nu} \frac{2 Z^{2}}{\left|\mathbf{R}_{\nu}\right|}=\alpha_{1} Z+\gamma_{\text {Ewald }} .
$$

Desta forma, a energia total por átomo será:

$$
\begin{array}{r}
E_{\text {tot }}=\frac{1}{N} \sum_{i} \varepsilon_{i}-\frac{1}{2} \Omega_{a t} \sum_{\mathbf{G} \neq \mathbf{0}} V_{\text {coul }}(\mathbf{G}) n(\mathbf{G})+\frac{1}{4} \Omega_{a t} \sum_{\mathbf{G}} \mu_{x c}(\mathbf{G}) n(\mathbf{G})+\alpha_{1} Z+ \\
+\gamma_{\text {Ewald }} . \quad(2.67)
\end{array}
$$


As funções de onda aqui utilizadas são representadas através de expansões de ondas planas. Para que estas expansões não fiquem muito complexas, o que tornaria o cálculo muito custoso, definiremos uma "Energia de Corte". Esta energia de corte nos impõe um limite para esta expansão, excluindo termos que tornem a energia cinética da função de onda $\left(\sum_{i, \mathbf{G}}\left|C\left(\mathbf{k}_{i}+\mathbf{G}\right)\right|^{2}\left(\mathbf{k}_{i}+\mathbf{G}\right)^{2}\right)$ maior do que ela. Além disso, no somatório sobre todos os pontos da rede recíproca, utilizaremos somente alguns pontos [9], ou mais especificamente, um ponto, o ponto $\Gamma$. Isto pode ser feito pois, como utilizamos células muito grandes, a respectiva zona de Brillouin será pequena e, desta forma, podemos dizer que o ponto $\Gamma$ representa bem o espaço recíproco.

O método descrito nesta seção é muito eficaz na determinação da energia do estado fundamental de um sistema. Entretanto, necessitamos ainda de um método eficaz para se obter a posição mais estável dos átomos.

A primeira idéia a respeito disso, foi implementada por Hellman e Feynmann [10]. Neste trabalho, Feynman acabou com um estereótipo que existia na época de que a energia era o único observável interessante de ser estudado. Feynman mostrou que as forças também eram fáceis de ser calculadas, uma vez que a energia fosse sabida.

Se dissermos que a força numa direção $x$ sobre uma partícula é dada por:

$$
\mathbf{F}_{x}=-\frac{\partial E}{\partial x}
$$

e sabendo que

$$
\frac{\partial E}{\partial x}=\frac{\partial}{\partial x} \int \psi^{*}(\mathbf{r}) H \psi(\mathbf{r}) d \mathbf{r},
$$

é fácil mostrar que a força será então dada por

$$
\mathbf{F}_{x}=-\int \psi^{*}(\mathbf{r}) \frac{\partial H}{\partial x} \psi(\mathbf{r}) d \mathbf{r}
$$


Desta forma obtêm-se as forças sobre os núcleos em todas as direções.

Car e Parrinello [11] também utilizaram conceitos clássicos em cálculos de estrutura eletrônica. Entretanto, eles foram além da idéia de Hellman e Feynman, utilizándo uma dinâmica clássica não somente para o movimento dos núcleos, como também para os orbitais.

Foi determinada então uma Lagrangeana para descrever a dinâmica do sistema constituído dos núcleos e dos orbitais:

$$
L=\sum_{i} \frac{1}{2} \mu \int_{\Omega} d \mathbf{r}\left|\dot{\psi}_{i}(\mathbf{r})\right|^{2}+\sum_{i} \frac{1}{2} M \dot{R}_{i}^{2}+\sum_{\nu} \frac{1}{2} \mu_{\nu} \dot{\alpha}_{\nu}^{2}-E\left[\psi_{i}, R_{i}, \alpha_{\nu}\right]
$$

onde $\mu$ e $\mu_{\nu}$ são massas fictícias, $M$ é a massa dos núcleos, $R$ representa a posição dos núcleos, $\psi$ é o orbital das equações de Kohn-Sham, $\alpha$ representa as condições de vínculo do sistema e $E$ é o potencial das equações de KohnSham (2.33).

Desta forma, as equações de movimento dos núcleos e dos orbitais serão descritas respectivamente por:

$$
\begin{aligned}
M_{i} R_{i} & =-\nabla_{R_{i}} E ; \\
\mu \ddot{\psi}_{i}(r, t) & =-\frac{\partial E}{\partial \psi_{i}^{*}(\mathbf{r}, t)}+\sum_{k} \lambda_{i k} \psi_{k}(\mathbf{r}, t),
\end{aligned}
$$

onde $\lambda_{i k}$ são multiplicadores de Lagrange.

Fazendo a dinâmica dos orbitais desta forma, economiza-se muito esforço, em função de não mais ser necessária a diagonalização de matrizes, como no esquema autoconsistente convencional. Apesar disto, este método foi sofrendo alterações, que o tornaram muito mais eficiente. Payne et al. [12] mudaram 2.73 a fim de torná-la mais eficiente, da seguinte maneira:

$$
\mu \ddot{\psi}_{i}(r, t)=-2 \gamma \dot{\psi}_{i}(\mathbf{r}, t)-\frac{\partial E}{\partial \psi_{i}^{*}(\mathbf{r}, t)}+\sum_{k} \lambda_{i k} \psi_{k}(\mathbf{r}, t)
$$


onde $\gamma$ é um termo de amortecimento.

A dinâmica para os núcleos implementada no programa[1] também sofreu algumas alterações em relação à descrição original de Car e Parrinello. A nova posição do íon é dada por

$$
\mathbf{R}_{i}^{n+1}=(1+\lambda) \mathbf{R}_{i}^{n}-\lambda \mathbf{R}_{i}^{n-1}+\frac{1}{M} \mathbf{F}_{i}^{n}
$$

onde $\lambda$ é um fator de amortecimento e $M$ é a massa dos núcleos. 


\section{Capítulo 3}

\section{A Superfície Si(100)}

O estudo de superfícies dos materiais é uma área de pesquisa relativamente recente, visto que ela teve um grande desenvolvimento somente nas últimas duas décadas. Antes disso, uma preocupação constante para os estudos de cristais era evitar os problemas de superfície, visto que se tratavam de problemas muito complexos. Nos últimos vinte anos, o desenvolvimento de técnicas e de computadores cada vez mais potentes possibilitou que os problemas deste tipo fossem tratados diretamente.

Neste capítulo descreveremos as principais características da superfície (100) do Silício. Esta é uma superfície já muito estudada [13], [14], [15], [16], em virtude de sua importância tanto na área de ciência básica como na área tecnológica.

Do ponto de vista tecnológico, esta superfície é utilizada para crescer alguns tipos de dispositivo. Existem outras superfícies muito importantes também para o crescimento de dispositivos. Podemos destacar aqui a superfície (111) do Silício, que é muito utilizada no crescimento de transistores bipolares. Já a superfície (100) é muito utilizada no crescimento de dispositivos do tipo MỌS (metal-óxido-semicondutor).

Esta superfície, como já dissemos, tem um papel muito importante também para a ciência básica. Tal importância vem do fato da relativa facilidade 
de se obterem grandes platôs nesta superfície, ou seja, a relativa facilidade de limpeza desta superfície. Além disso, esta é uma superfície muito fácil de ser descrita teoricamente, como vamos mostrar adiante.

Passaremos agora a descrever as principais características desta superfície, métodos de obtenção e descrição e as principais reconstruçôes.

\subsection{Preparação da Superfície}

A preparação da superfície é um ítem muito importante, em virtude de que para medidas precisas como as que vamos tratar, são necessárias superfícies com níveis de impureza baixíssimos. Apesar do nosso trabalho não estar voltado especificamente para este assunto, vamos dar uma pequena noção de métodos utilizados para a preparação da superfície (100) do Silício.

O primeiro passo é cortar um cristal de Si ao longo do plano (100). A superfície obtida através deste corte é então tratada, a fim de torná-la mais homogênea possível. Para evitar a presença de impurezas, o tratamento deve ser realizado em câmaras de ultra alto vácuo. Existem dois processos muito usados para proceder a limpeza desta superfície. No primeiro, a superfície é tratada através de reações químicas [17], que eliminam as impurezas e imperfeições desta. Outro método é através de tratamentos térmicos [16]. O primeiro método é muito mais eficiente do que o segundo, apesar de ambos normalmente serem usados contiguamente. Apesar deste tratamento, é impossível obtermos uma superfície totalmente perfeita. Sempre existirão sobre ela degraus ou outros defeitos como vacâncias e impurezas. Mas como as amostras são geralmente grandes, sempre podem ser obtidos platôs da ordem de centenas de Angstrons, o que é o suficiente para o crescimento de pequenas estruturas.

Do ponto de vista teórico, descrevemos a superfície utilizando uma supercélula a qual são aplicadas condições periódicas de contorno. O tamanho 
Como foi descrito na seção anterior, quando nos referimos à superfície (100) do Silício, supomos uma superfície formada por um corte ao longo do plano cristalino (100) de um cristal formado por uma rede cúbica de face centrada, que é a rede de Bravais do Silício. Teremos exposta então, nesta face, a superfície com a qual iremos trabalhar. Uma superfície obtida desta maneira é estável para alguns materiais, entre eles a maioria dos metais, mas para os semicondutores, esta afirmação não é válida.

Após o corte, ocorre um processo de reconstrução [18] , onde os átomos da superfície se reorganizam em posições mais estáveis, saturando ao máximo as ligações livres provocadas pelo corte.

Na Figura 3.2 apresentamos esquematicamente o que ocorre com os átomos da superfície e com as ligações flutuantes durante a reconstrução. Assim que a superfície é 'cortada', os átomos de Si ainda estarão na sua posição cristalina (fcc), com duas ligações flutuantes cada um, devido a ausência de dois átomos para completar a hibridização $s p^{3}$ do Si (Figura 3.2a). Esta é uma estrutura extremamente instável devido a densidade de ligações flutuantes na superfície. Para diminuir esta instabilidade, os átomos da superfície se agrupam em dímeros, com uma ligação covalente entre os átomos, reduzindo assim a densidade de ligações flutuantes pela metade (Figura 3.2b). Com isto, a distância entre os átomos diminuirá e, consequentemente, a distância entre as ligações flutuantes destes átomos também diminuirá. Isto facilita a

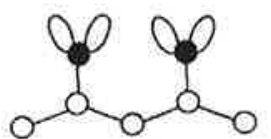

(a)

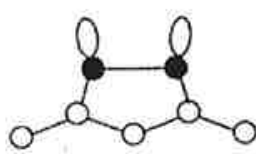

(b)

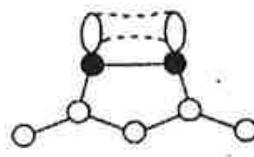

(c)

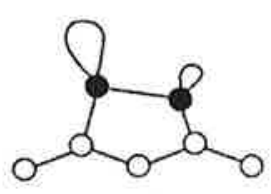

(d)

Figura 3.2: Reorganização dos orbitais durante a reconstrução da superfície $\mathrm{Si}(100)$. 


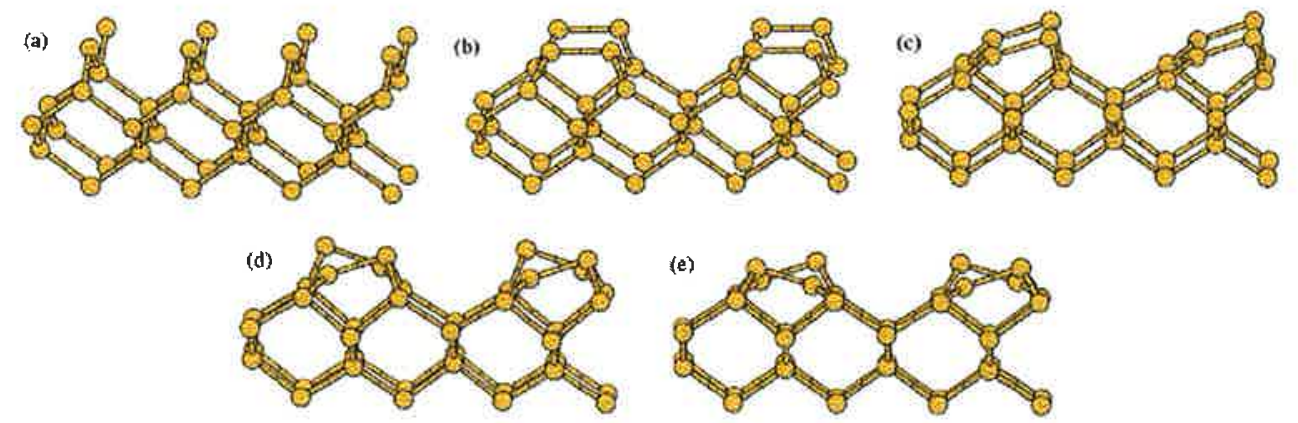

Figura 3.3: Esquema das reconstruções da superfície (100) do Silício: (a) superfície

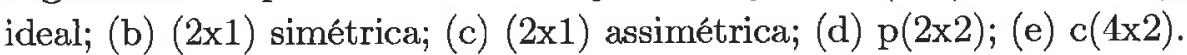

formação de uma ligação $\pi$ entre estes, envolvendo as duas ligações flutuantes restantes (Figura 3.2c). Como os átomos estão mais próximos agora, ocorre uma transferência de carga de um átomo do dímero para o outro átomo, de forma que o átomo que recebe a carga irá ficar numa posição superior em relação ao que cede a carga, formando assim um deslocamento no dímero (Figura 3.2d).

Geometricamente, a reconstrução da superfície acarreta também numa perda de simetria. Isto pode ser visto na sequência esquematizada na Figura 3.3.

Na Figura 3.3a, observamos a superfície ideal, que seria obtida logo após o corte do cristal. Como já foi mencionado anteriormente, a tendência é que se formem dímeros sobre a superfície, sendo que estes se agrupam em colunas, como pode ser visto na Figura $3.3 \mathrm{~b}$. Esta reconstrução é chamada de $(2 \times 1)$ simétrica. $O$ nome vem do fato da célula unitária bidimensional da superfície ser constituída por dois átomos numa direção $i$ e um átomo numa direção ortogonal $j$. A relaxação dos átomos da superfície de forma que os átomos dos dímeros fiquem deslocados é mostrada na Figura 3.3c. Novamente a célula unitária bidimensional é formada por dois átomos, mas esta reconstrução é chamada (2x1) assimétrica, em virtude do deslocamento 
dos dímeros. Cálculos teóricos e resultados de Microscopia de Tunelamento Eletrônico (STM) e Low Emission Electron Diffraction (LEED) mostraram que existiam outras reconstruções mais estáveis que a (2x1) assimétrica. Se, ao invés dos dímeros da superfície ficarem todos virados para o mesmo lado, eles ficarem dispostos de maneira que dímeros vizinhos numa mesma coluna fiquem deslocados no sentido contrário, teremos uma nova reconstrução, chamada de (2x2), como pode ser visto na Figura 3.3d. Nesta reconstrução a célula unitária bidimensional contém dois átomos em cada direção. Apesar disso, a reconstrução da superfície (100) do Silício tida como mais estável é a (4x2). Esta reconstrução pode ser observada na Figura 3.3e. Ela é obtida invertendo-se o deslocamento de dímeros em colunas diferentes, de forma que os primeiros dímeros vizinhos em ambas as direções estarão deslocados em sentidos diferentes. Neste caso, a célula unitária bidimensional é formada por quatro átomos numa direção e dois na outra.

Todas estas reconstruções já foram exaustivamente estudadas [14], [16], [15], [13], e pode-se dizer que o ganho de energia para cada uma já é bem definido. Nós fizemos alguns cálculos, no sentido de obter o ganho de energia para cada reconstrução. Utilizamos para estes cálculos uma célula 4x2, com 6 camadas de Si e 5 camadas de vácuo (metade da célula descrita na Figura 3.1). A densidade eletrônica total foi calculada com 8 pontos especiais e a base de ondas planas foi expandida até uma energia de 20Ry.

Com a dimerização da superfície, obtivemos um ganho de energia de $1.60 \mathrm{eV}$ por dímero. Com a relaxação para uma estrutura $(2 \times 1)$ asimétrica, obtivemos um ganho de $0.14 \mathrm{eV}$ por dímero. Com a mudança para uma

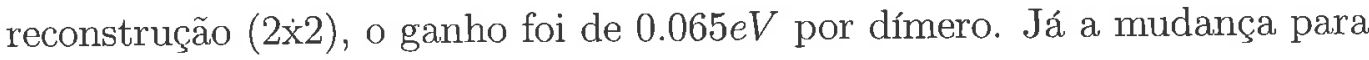
a reconstrução mais estável $(4 \times 2)$, resultou num ganho de energia, em relação a reconstrução $(2 \times 2)$ de somente $0.005 \mathrm{eV}$ por dímero. Os valores reportados na literatura concordam muito bem com nossos resultados. Os resultados mais aceitos [14] para os ganhos de energia são $1.8 \pm 0.1 \mathrm{eV} /$ dímero para a dimerização, $0.12 \pm .01 \mathrm{eV} /$ dímero para a relaxação para uma estrutura $(2 \mathrm{x} 1)$ 
Tabela 3.1: Distância entre átomos dos dímeros e ângulos de deslocamento, como esquematizado na Figura 3.4. As distâncias são dadas em ângstrons e os ângulos em graus.

\begin{tabular}{|c|c|c|}
\hline & Distância $(\mathrm{d})(\AA)$ & Ângulo $(\omega)\left(^{\circ}\right)$ \\
\hline Ideal & 3.85 & - \\
\hline$(2 \times 1)$ simétrica & 2.30 & 0 \\
\hline$(2 \times 1)$ assimétrica & 2.26 & 18.4 \\
\hline$(2 \times 2)$ & 2.32 & 18.2 \\
\hline$(4 \times 2)$ & 2.24 & 17.7 \\
\hline
\end{tabular}

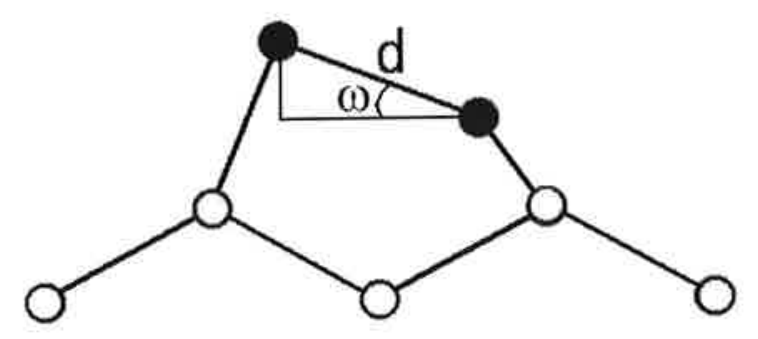

Figura 3.4: Forma como o ângulo $(\omega)$ e a distância (d) são medidos.

asimétrica, $0.048 \pm .018 \mathrm{eV} /$ dímero para a passagem para uma reconstrução (2x2) e $0.003 \pm .013 \mathrm{eV} /$ dímero para se obter a reconstrução mais estável $(4 \times 2)$.

Além dos ganhos de energia, foi feito um estudo das propriedades estruturais de cada reconstrução, onde observamos o ângulo de deslocamento dos dímeros e a distância de ligação entre os átomos dos dímeros da superfície. Estes resultados podem ser observados na Tabela 3.1. Observa-se que o ângulo médio de deslocamento dos dímeros é de $18.1^{\circ}$ e a distância média entre átomos vale $2.28 \AA$. Estes valores também concordam muito bem com resultados preliminares[14], onde a distância média entre os átomos dos dímeros vale $2.27 \AA ̊$ e o ângulo médio é de $18.9^{\circ}$. A forma como o ângulo e a distância 
são medidos está esquematizada na Figura 3.4. 


\section{Capítulo 4}

\section{Crescimento de Ge Sobre $\operatorname{Si}(100)$}

O nosso trabalho enfocará basicamente o crescimento de Germânio sobre a superfície (100) do Silício. Este tipo de heteroestruturas são muito importantes, pois nos fornecem informações sobre a interface Ge-Si, além de poderem dar origem a dispositivos, que supostamente seriam mais rápidos e eficientes.

O perfeito entendimento de como estas estruturas crescem é muito importante pois fornecerá meios de promover um crescimento perfeito destas heteroestruturas, reduzindo ao mínimo o número de defeitos presentes. O nosso trabalho, entretanto, dará um enfoque mais microscópico ao tema. A maneira como os primeiros átomos adsorvidos se organizam e difundem ao chegarem à superfície ainda não é bem entendida. Portanto, iremos nos preocupar com os primeiros estágios do crescimento, ou seja, estudaremos apenas estruturas muito pequenas, com 1, 2 ou 3 átomos adsorvidos. $O$ conhecimento destas pequenas estruturas é imprescindível para o entendimento do crescimento.

Neste capítulo, faremos uma breve revisão de alguns dos resultados mais significativos, tanto teóricos como experimentais, para a adsorção de peque- 
nas estruturas de Ge e Si sobre a superfície Si(100). Após isto, apresentaremos alguns resultados obtidos por nós para o estudo de pequenas estruturas de Ge sobre $\mathrm{Si}(100)$, bem como algumas imagens teóricas de STM destas estruturas.

\subsection{Revisão Bibliográfica}

Um dos trabalhos mais citados que utilizam métodos ab initio no estudo da adsorção de átomos sobre a superfície Si(100) é o de Brocks, Kelly e Car[19], de 1991. Neste trabalho foram estudadas as posições mais estáveis para a adsorção de átomos de Si sobre a superfície. Como resultado, eles obtiveram um mapeamento energético da superfície para a adsorção de Si. Após isto, começaram a se proliferar resultados para vários tipos de átomos e para vários tamanhos de estruturas adsorvidas. Apesar do nosso trabalho ter um enfoque para a adsorção de Germânio, daremos importância também para a adsorção de $\mathrm{Si}$, em virtude de existirem muito mais resultados para $\mathrm{Si}$ sobre $\mathrm{Si}(100)$ do que para Ge sobre $\mathrm{Si}(100)$. A comparação entre estruturas formadas pelos dois tipos de átomos é imediata, em virtude de se tratarem de elementos com o mesmo número de elétrons de valência, a mesma estrutura cristalina, enfim, por terem um comportamento estrutural muito semelhante.

A primeria semelhança começa para as posições ligantes de um único átomo sobre a superfície. Para o $\mathrm{Si}$, este estudo ${ }^{1}$ foi realizado por Brocks, Kelly e Car [19] e por Yamasaki, Uda e Terakura [22], enquanto que para o Ge, o estudo foi feito por Milman et al. [23]. Todos os estudos encontram a mesma posição como sendo a mais estável, o sítio entitulado M. Tal sítio pode ser visto na Figura4.1.

Entretanto, ocorrem algumas discrepâncias entre resultados para a ad-

\footnotetext{
${ }^{1}$ Todos os trabalhos relatados aqui foram feitos através de métodos ab initio. Existem vários resultados obtidos através de métodos semiempíricos, porém, todos reportam posições ligantes diferentes dos cálculos $a b$ initio. Para referências, ver [20][21].
} 


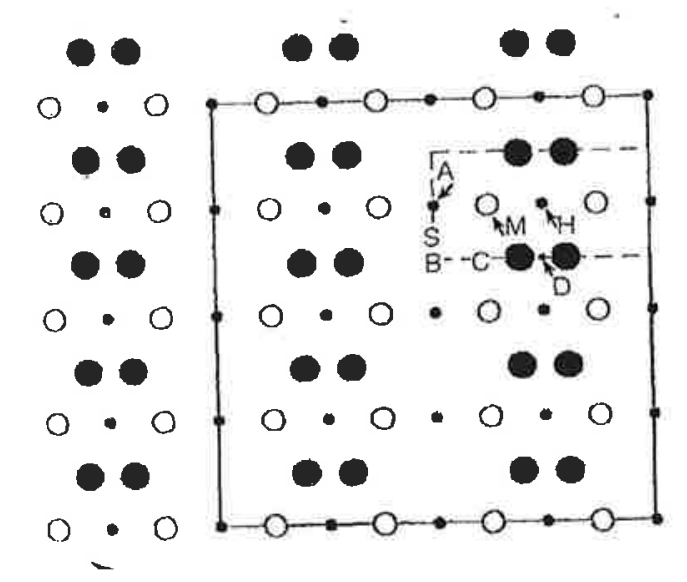

Figura 4.1: Posições ligantes para um átomo sobre a superfície (100) do Silício. Os círculos cheios representam os dímeros de Si da primeira camada, os abertos representam os átomos da segunda camada e os círculos pequenos representam os átomos da terceira camada.

sorção de Si. Os trabalhos de Brocks [19] e Yamasaki [22] encontram diferentes disposições para os dímeros da superfície quando um átomo é adsorvido no sítio M. No trabalho de Brocks, Kelly e Car, os dímeros da superfície mais próximos do átómo adsorvido têm o seu deslocamento de forma que o átomo mais próximo do átomo adsorvido fique numa posição superior ao outro átomo do dímero. Entretanto, a relaxação dos dímeros para esta posição se deve ao fato de Brocks et al. terem usado para o seu cálculo uma célula reduzida $(2 \times 1)$. Um estudo semelhante foi feito por Yamasaki et al., mas utilizando uma célula maior $(4 \mathrm{x} 4)$, como a descrita por nós na Figura 3.1. Eles obtiveram que as duas colunas de dímeros permanecem inalteradas em relação à direção do deslocamento dos dímeros. A única mudança é em relação ao ângulo do deslocamento dos dímeros ligados ao átomo adsorvido, que ficam muito menores.

A organização dos átomos da superfície quando um átomo de Germânio é adsorvido pela superfície é diferente das duas situações descritas anteriormente. Milman et al. [23] também utilizaram uma célula $(4 \times 4)$ e encontraram 
que os dímeros mais próximos ao átomo de Ge, nas duas colunas de dímeros, ficariam com o átomo mais próximo do Ge para baixo, mas com um ângulo de deslocamento entre os átomos do dímero muito pequeno.

No caso dos estudos feitos por Brocks et al. e Milman et al. , eles relatam que os átomos se ligam somente aos átomos dos dímeros mais próximos. Não é observada nenhuma ligação com átomos da segunda camada. O trabalho de Uda et al. não menciona nada a respeito.

O segundo mínimo de energia obtido por todos os estudos é o sítio $\mathbf{H}$. Este sítio é descrito por Brocks et al. como estando sobre uma coluna de dímeros, numa posição central entre dois dímeros. Novamente, esta descrição se deve ao fato de eles terem usado uma célula pequena, o que força uma simetria errada ao sistema. Em outros trabalhos, este sítio ligante é um pouco deslocado da posição central. Este átomo se liga com os quatro átomos mais próximos. Um detalhe interessante é que no resultado de Milman, todos dímeros da coluna onde o átomo se encontra adsorvido se apresentavam simétricos, ou sem nenhum deslocamento.

O mapeamento da superfície energética para a adsorção de Si e Ge sobre Si(100) nos fornece uma idéia das barreiras de difusão e dos caminhos mais prováveis para a difusão dos átomos sobre a superfície. Para o Si, um dos caminhos com a menor barreira de difusão é ao longo das colunas de dímeros, percorrendo o caminho $\mathbf{M} \rightarrow \mathbf{H} \rightarrow \mathbf{D}$. A barreira de difusão por este caminho foi calculada como sendo $0.6 \mathrm{eV}$. Existe ainda um caminho perpendicular às colunas de dímeros, passando pelo sítio B. Esta barreira vale $1.0 \mathrm{eV}$. Estes valores mostram claramente a anisotropia presente sobre a superfície $\mathrm{Si}(100)$, o que influencia muito no processo de crescimento. Para o Germânio, as barreiras encontradas são da mesma ordem das barreiras para o Silício, porém o caminho para a difusão é sensivelmente diferente. Para difusão ao longo das cadeias de dímeros, o caminho mais provável vai do sítio $\mathbf{M}$ ao sítio $\mathrm{D}$, sem passagem necessária pelo sítio $\mathbf{H}$. Esta barreira vale $0.62 \mathrm{eV}$. Numa difusão perpendicular às colunas de dímeros o átomo passaria pelo sítio $\mathbf{S}$ e teria que 
transpor uma barreira de $0.95 \mathrm{eV}$.

Em virtude destes átomos possuírem uma taxa de difusão muito grande, eles são estruturas muito móveis à temperatura ambiente, não sendo possível a observação destas estruturas isoladas através de STM. Entretanto, quando dois átomos se juntam sobre a superfície formando um dímero, eles podem ser visualisados através desta técnica. Isto torna o estudo de dímeros muito atrativo por causa da possibilidade de comparação de resultados experimentais com resultados teóricos. Isto faz com que trabalhos sobre dímeros adsorvidos sobre a superfície sejam muito abundantes [24][25][26][27]. Outra importância do estudo de dímeros está no fato dos dímeros serem a menor estrutura estável presente na superfície a temperatura ambiente. Portanto, eles também são muito importantes no processo de crescimento.

Existem somente quatro estruturas estáveis para dímeros de Si sobre $\mathrm{Si}(100)$. Tais estruturas podem ser vistas na Figura 4.2. O dímero mais estável encontrado foi o $\mathbf{A}$, seguido por $\mathbf{B}, \mathbf{C}$ e $\mathbf{D}$, com energias relativas de $0.0,0.08,0.18$ e $0.76 \mathrm{eV}$ respectivamente [26].

Resultados experimentais de STM [28] mostram que a estrutura D nunca é observada de forma isolada. Este resultado concorda com resultados teóricos, que afirmam que esta é a estrutura menos estável entre as estudadas.

Entre as estruturas mais estáveis (A e B) observou-se uma razão de $10: 1$ no número de dímeros A:B. Através desta proporção, Wingerden et al. estimou a diferença de energia entre as estruturas $\mathbf{B}$ e $\mathbf{A}$ como sendo $60 \mathrm{meV}$. Este resultado está de acordo com os resultados teóricos[22].

Apesar das estruturas A e B serem as mais estáveis, a estrutura mais abundante durante o crescimento epitaxial, à temperatura ambiente, de Si sobre $\mathrm{Si}(100)$ através de MBE é o dímero C. De todos os dímeros presentes na superfície, $67 \%$ são do tipo C. Entretanto, isto não quer dizer que o dímero $\mathbf{C}$ seja o mais estável. Supõe-se que ele seja o mais abundante por um processo cinético. Isto pode ser visualizado se tomarmos um átomo adsorvido num sítio mais estável $\mathbf{M}$ e se considerarmos as três colunas de dímeros da 


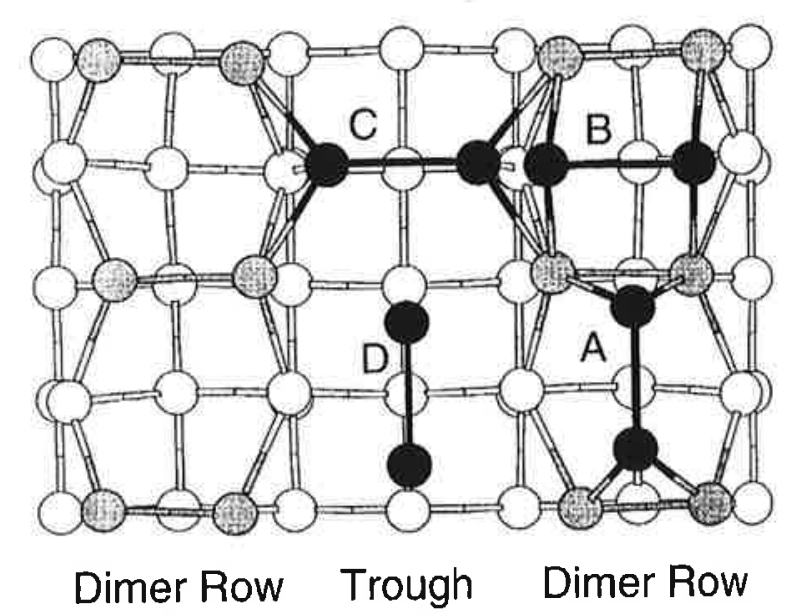

Figura 4.2: Estruturas mais estáveis de dímeros sobre a superfície. Os círculos pretos representam os dímeros adsorvidos; os círculos em cinza representam os dímeros da superfície e os outros círculos representam átomos mais profundos.

superfície mais próximas dele. Se o próximo átomo que chegar à superfície cair sobre a coluna a qual o átomo $\mathbf{M}$ está ligado, ele irá difundir sobre a coluna de dímeros até se aproximar do átomo M. Da ligação destes átomos será formado um dímero A ou B. Já se o átomo cair nas outras colunas de dímeros, ele difundirá até se ligar ao átomo $\mathbf{M}$ e formará um dímero $\mathbf{C}$. Isto nos dá uma razão de $2 / 3$ de chances de se formarem dímeros C. Assim, fica explicada a diferença de população entre estes dímeros.

Apesar dos dímeros serem ilhas estáveis, existem muitos estudos relacionados à difusão de dímeros sobre a superfície. Os resultados reforçam o fato da anisotropia da superfície (100) do Silício. Mais uma vez fica evidente que a difuisão na direção das colunas de dímeros é muito mais fácil do que numa direção perpendicular a estas. Também observou-se que a difusão no topo das colunas de dímeros é muito mais fácil do que entre elas [29]. Estima-se a barreira para difusão de um dímero B em cima de uma coluna de dímeros como sendo $1.08 \mathrm{eV}$, enquanto que a barreira de difusão de um 
dímero $\mathbf{C}$ entre duas colunas de dímeros vale $1.28 \mathrm{eV}$. Estas barreiras são referentes à difusão fracionada do dímero, ou seja, os átomos constituintes do dímero se separam, difundem e depois voltam a se juntar. Estes processos são relevantes em virtude de ocorrerem à temperaturas relativamente baixas $(\sim 400 K)$, contrário à difusão dos dímeros inteiros. Outro processo interessante de ser estudado é a transição entre os dímeros $\mathbf{A}$ e $\mathbf{B}$, uma vez que os estudos teóricos mostram que estes dímeros são quase degenerados. Borovsky et al. [29] estimaram esta barreira como sendo de $0.70 \mathrm{eV}$. Também foi estimada neste trabalho a barreira para difusão de um dímero da posição B para a posição $\mathbf{C}$, resultando em $1.36 \mathrm{eV}$. Assim fica provado que a difusão ao longo das colunas de dímeros envolve barreiras muito menores do que para direções perpendiculares a estas.

Para dímeros de Ge sobre $\mathrm{Si}(100)$ existem poucos resultados disponíveis na literatura. Um resultado muito interessante foi obtido por Qin e Lagally[25], onde eles fizeram um estudo via STM da formação de dímeros. Foi observado que os dímeros $\mathbf{C}$ apresentavam um sinal diferente para as imagens cheias e vazias. Nas imagens cheias, observou-se que o dímero tinha um sinal muito fraco, o que os levou a dizer que na verdade não se tratava de um dímero, e sim urn par de átomos. Esta estrutura foi considerada como sendo de vital importância para a formação de estruturas maiores. Especula-se que durante a formação do dímero $\mathbf{C}$, como descrito anteriormente, formese primeiramente uma estrutura metaestável composta por dois átomos em posições $\mathbf{M}$ vizinhas, para posterior formação do dímero $\mathbf{C}$.

Em virtude da população de dímeros sobre a superfície ser determinada por fatores cinéticos, o estudo de estruturas maiores adsorvidas se torna mais complexo. Se a formação de dímeros já é determinada cineticamente, a formação de estruturas maiores também deveria ser. Entretanto, estudos experimentais [28] mostram que somente uma estrutura de três átomos é observada no crescimento de $\mathrm{Si}$ sobre $\mathrm{Si}(100)$. Esta estrutura é composta por um dímero $\mathbf{C}$ mais um átomo num sítio $\mathbf{M}$ vizinho. Esta estrutura pode ser 


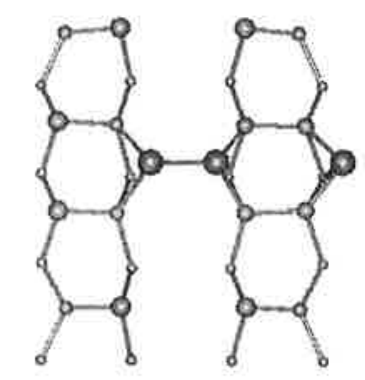

Figura 4.3: Única estrutura com três átomos adsorvidos observada experimentalmente.Os círculos grandes representam os átomos adsorvidos.

visualizada na Figura4.3.

O fato desta estrutura ser a única observada está de acordo com estudos teóricos, que dizem que esta estrutura é a energeticamente mais favorável[22].

Wingerden et al. observaram duas formas distintas para a formação desta estrutura. Na primeira, o trímero é formado pela incorporação direta de um monômero a um dímero $\mathrm{C}$ através de difusão ao longo das cadeias de dímeros. Este processo pode ser observado pois os dímeros $\mathrm{C}$ são vistos como uma mancha muito fraca em imagens de STM dos orbitais ocupados. Quando um terceiro átomo é incorporado, este dímero se torna mais visível nestas imagens. O que se pode observar então foi uma repentina nitidez do dímero. O átomo incorporado no ponto $\mathrm{M}$ não é visto em imagens cheias, como já havia sido mostrado por Swartzentruber [30]. Um outro meio para este trímero se formar é através de um dímero A ou B. Este processo envolve a quebra da ligação do dímero e uma posterior adsorção de um terceiro átomo. Ambos os processos descritos acima ocorrem com a mesma probabilidade.

Os trímeros são estruturas interessantes de serem estudadas pelo fato de serem o estado de transição inicial do crescimento, partindo de dímeros, que são as menores ilhas estáveis, e indo em direção a estruturas maiores adsorvidas. Assim como o trímero da Figura 4.3, estruturas maiores de $\mathrm{Si}$, 
contendo vários átomos, também tendem a se organizar em linha, na direção [110].

Este tipo de estruturas são muito bem ordenadas, e elas podem ser observadas em imagens de STM no crescimento de amostras que possuem de graus. Este tipo de amostras possuem uma característica diferente, uma vez que as colunas de dímeros da superfície são perpendiculares umas às outras em degraus diferentes. Isto faz com que as estruturas cresçam em direções diferentes em diferentes degraus[31]. Este fato nos fornece novamente uma boa demonstração da anisotropia existente na superfície, mostrando como esta anisotropia influencia no crescimento.

\subsection{Resultados}

A partir de agora, passaremos a descrever os resultados obtidos por nós [32] para a adsorção de monômeros, dímeros e trímeros sobre a superfície $\operatorname{Si}(100)$.

Como descrito no Capítulo 2, para realizar tais cálculos, utilizamos um método de primeiros princípios, baseado na Teoria do Funcional da Densidade, dentro da Aproximação da Densidade Local para os termos de correlação e Troca. A interação íon-elétrons foi descrita através de Pseudopotenciais de Norma Conservada do tipo BHS com a separação de KleinmanBylander. Utilizamos uma expansão em ondas planas até uma energia de corte de 12Ry. A Zona de Brillouin foi simulada através de um ponto especial $(\Gamma)$, salvo algumas excessões, às quais explicitaremos posteriormente.

Para simular a superfície, utilizamos uma supercélula, como a descrita na Figura 3.1. Todos os átomos foram relaxados, exceto a última camada de Si e os átomos de Hidrogênio. As estruturas eram consideradas relaxadas quando a forças sobre todos os átomos, em todas as componentes cartesianas, eram menores do que 0.001a.u.. 


\subsubsection{Monômeros}

Para a adsorção de um átomo de Germânio sobre a superfície, foram estudadas duas configurações, as duas mais estáveis, que, como já foi mencionado anteriormente, são os sítios $\mathbf{M}$ e $\mathbf{H}$.

A simulação foi feita da seguinte maneira: foi tomada uma célula $4 x 4 \mathrm{com}$ reconstrução $(4 x 2)$ e foi posto um átomo na posição $\mathbf{M}(\mathbf{H})$ a uma distância de $2 \AA$ da superfície. A partir desta estrutura inicial, foi deixado que o sistema relaxasse e que o átomo adsorvido fosse para uma posição ligante de mínima energia. Entretanto, foram encontradas estruturas diferentes das descritas por Milman.

As diferenças se referem principalmente ao sentido do deslocamento dos dímeros de Silício da superfície. Para o sítio $\mathbf{M}$, encontramos que o deslocamento dos dímeros que estão ligados ao átomo de Germânio estão fora de fase, ou com o deslocamento invertido. Além disto, este átomo não se encontra exatamente sobre o átomo de Si da segunda camada, e sim a uma distância de $0.86 \AA$ desta posição, no sentido da outra coluna de dímeros. Para o sítio $\mathbf{H}$, encontramos que o deslocamento dos dímeros da superfície ficam no mesmo sentido do deslocamento dos dímeros na reconstrução (4x2). A diferença está no ângulo destes deslocamentos, como pode ser visto na Tabela 4.1, que são muito menores. Além disto, a posição do átomo não é exatamente no centro de dois dímeros da superfície, e sim um pouco deslocado, como pode ser visto na Figura 4.4.

Em virtude dos resultados diferentes dos descritos por Milman[23], realizamos uma nova simulação, mudando as posições iniciais dos átomos. Os dímeros da superfície foram colocados nas posições descritas por Milman. Inicialmente, o átomo de Germânio adsorvido foi mantido fixo, e os átomos de Si foram movidos. Quando as forças sobre estes estavam menores do que o critério de convergência, o átomo adsorvido foi solto. Desta forma foi possível encontrar o sítio $\mathbf{M}$, descrito por Milman. Como obtivemos duas 

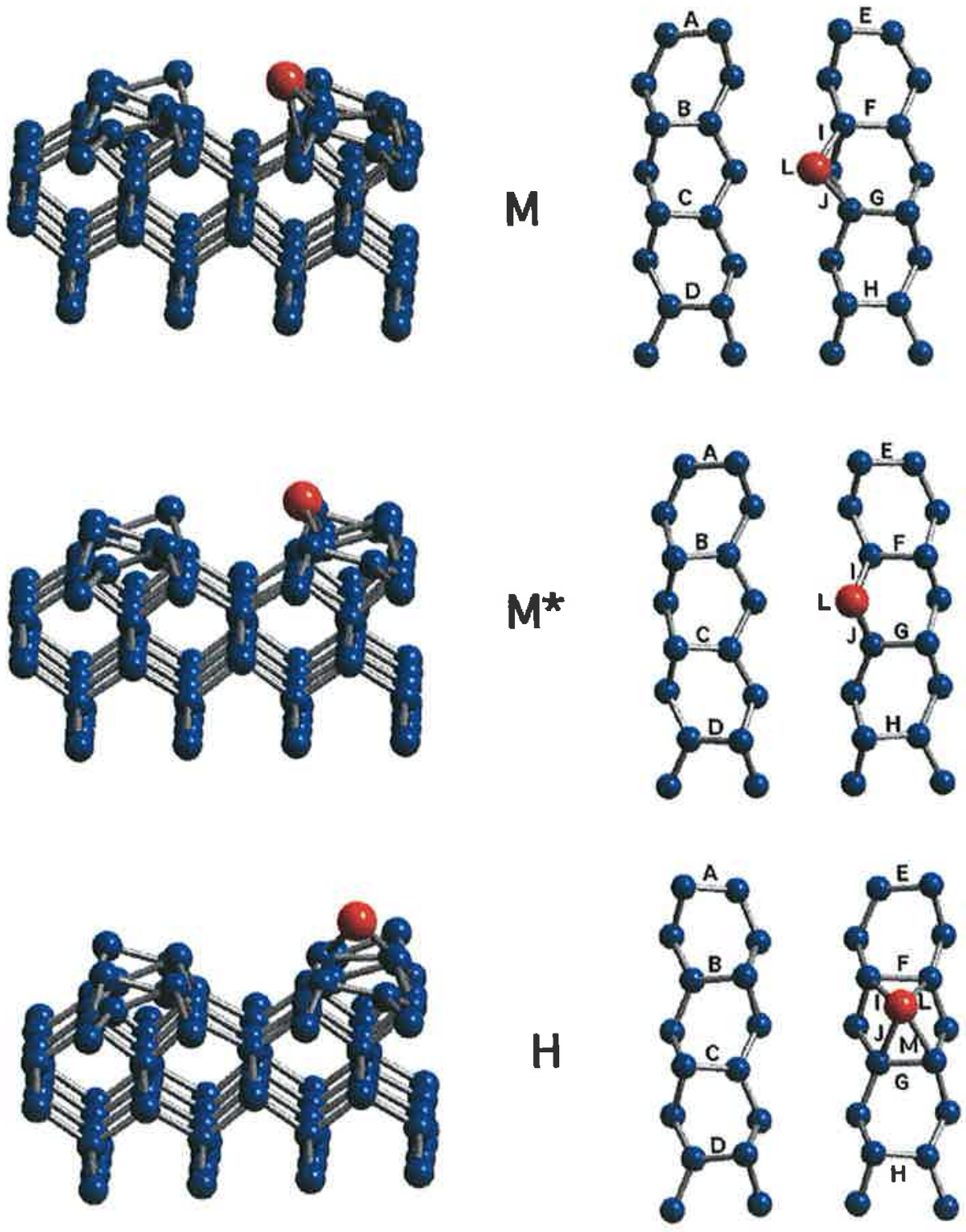

Figura 4.4: Imagens das três estruturas estudadas para a adsorção de um átomo de Ge sobre a superfície. As imagens da direita nos fornecem uma visão superior da estrutura. As letras servem de referência para as distancias mostradas na Tabela4.1. Para os sítios $\mathbf{M}$ e $\mathbf{M}^{*}$, a letra $\mathrm{L}$ se refere à distância do átomo de $\mathrm{Ge}$ ao átomo de $\mathrm{Si}$ da segunda camada. 
Tabela 4.1: Ângulos de deslocamento de dímeros da superfície e distâncias entre átomos para monômeros adsorvidos. As letras estão indicadas na Figura 4.4. As distâncias são dadas em ângstrons e os ângulos em graus.

\begin{tabular}{|c|c|c|c|c|c|c|}
\hline \multirow{2}{*}{} & \multicolumn{2}{|c|}{$\mathbf{M}$} & \multicolumn{2}{c|}{$\mathbf{M}^{*}$} & \multicolumn{2}{c|}{$\mathbf{H}$} \\
\cline { 2 - 7 } & dist. $(\AA)$ & Angulo $\left(^{\circ}\right)$ & dist. $(\AA)$ & Angulo $\left(^{\circ}\right)$ & dist. $(\AA)$ & Ângulo $\left({ }^{\circ}\right)$ \\
\hline A & 2.29 & 18.7 & 2.30 & 18.6 & 2.32 & 19.2 \\
\hline B & 2.30 & 18.8 & 2.30 & 18.7 & 2.31 & 19.2 \\
\hline C & 2.30 & 18.6 & 2.30 & 18.7 & 2.31 & 18.9 \\
\hline D & 2.29 & 18.6 & 2.30 & 18.6 & 2.32 & 19.2 \\
\hline E & 2.28 & 18.8 & 2.28 & 16.3 & 2.31 & 18.3 \\
\hline F & 2.34 & 15.1 & 2.39 & 14.8 & 2.55 & 4.0 \\
\hline G & 2.39 & 8.6 & 2.39 & 14.8 & 2.38 & 16.5 \\
\hline H & 2.26 & 14.4 & 2.28 & 16.5 & 2.30 & 17.3 \\
\hline I & 2.44 & - & 2.43 & - & 2.45 & - \\
\hline J & 2.43 & - & 2.43 & - & 3.07 & - \\
\hline L & 2.49 & - & 2.61 & - & 2.38 & - \\
\hline M & - & - & - & - & 2.77 & - \\
\hline
\end{tabular}

configurações distintas que podem ser consideradas como sendo o sítio $\mathbf{M}$, chamaremos esta última de $\mathbf{M}^{*}$. Utilizamos a mesma técnica para tentar obter o sítio $\mathrm{H}$ descrito por Milman, mas não foi possível.

As estruturas encontradas por nós podem ser vistas na Figura 4.4. As distâncias entre os átomos dos dímeros da superfície e do átomo de Germânio em relação aos átomos de Si próximos podem ser vistos na Tabela 4.1. Os ângulos de deslocamento dos dímeros também podem ser vistos nesta tabela.

Energeticamente, a estrutura mais estável encontrada foi o sítio M, seguido do sítio $\mathbf{H}$ e do sítio $\mathbf{M}^{*}$. As diferenças de energia relativa ao sítio mais estável $\mathbf{M}$ são, respectivamente 0.0, 0.32 e $0.47 \mathrm{eV}$. Em virtude dos resultados encontrados por nós estarem contrastantes em relação aos resultados de Milman, resolvemos refazer os cálculos, mas aumentando a precisão através do aumento do número de pontos especiais para o cálculo da densidade eletrônica. Os resultados obtidos com quatro pontos especiais nos fornece a mesma ordenação para as estruturas mais estáveis. As diferenças energéticas en- 

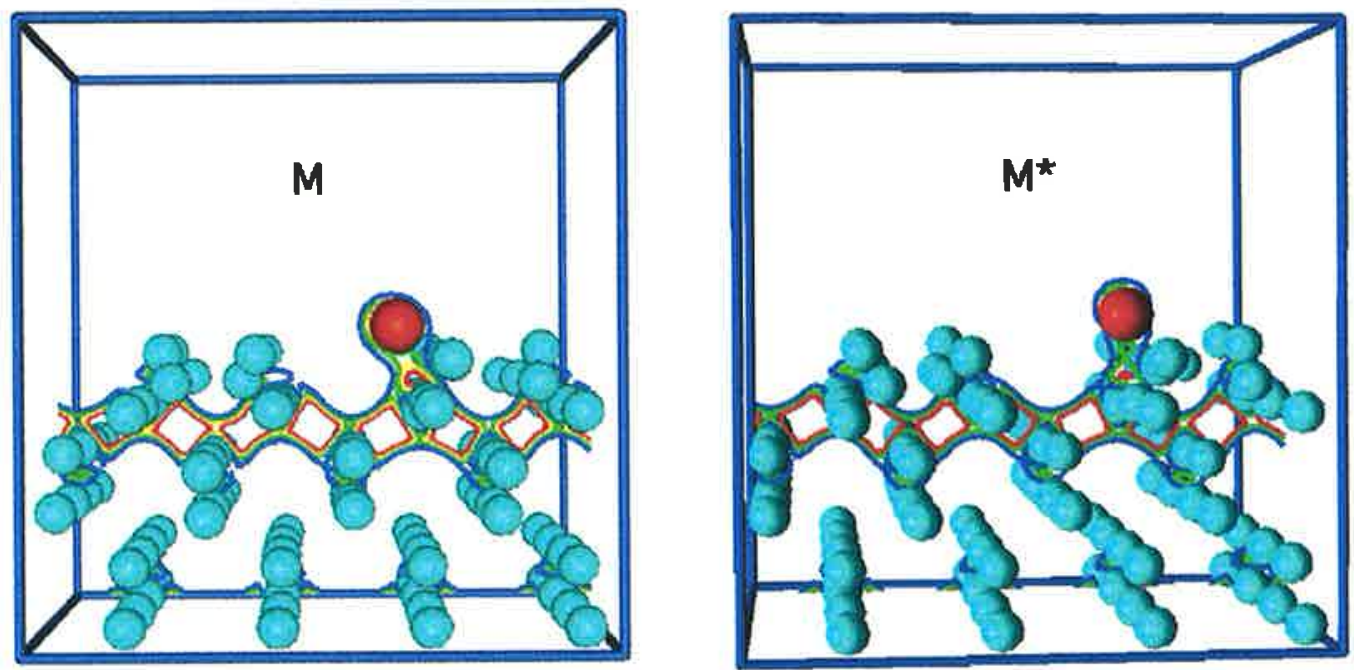

Figura 4.5: Distribuição de densidade de carga total para os sítios $\mathbf{M}$ e $\mathbf{M}^{*}$. Note que para o sítio $\mathbf{M}$, existe uma tendência á formação de uma ligação entre o átomo de Ge (vermelho) e o átomo de $\mathrm{Si}$ da segunda camada. As linhas isoenergéticas vermelhas indicam uma maior densidade de carga e as azuis uma menor densidade.

contradas foram, respectivamente, $0.0,0.10$ e $0.38 \mathrm{eV}$. Apesar da diferença das energias relativas, as geometrias podem ser consideradas como convergidas com um ponto especial, visto que as posiçôes mudaram menos de $0.02 \AA$ quando as estruturas foram recalculadas com quatro pontos especiais. Além disso, observou-se o mesmo comportamento para as distribuições de carga para os dois níveis de cálculo.

As posições encontradas por nós como sendo as mais estáveis se assemelham muito com os resultados obtidos por Yamasaki [22] para adsorção de Si sobre $\mathrm{Si}(100)$. Se fizermos uma comparação entre os resultados obtidos por Yamasaki com outros trabalhos para adsorção de Si sobre $\mathrm{Si}(100)$, podemos dizer que o primeiro é o mais preciso, em função do tamanho da célula utilizada.

Observando as distâncias atômicas para os sítios $\mathbf{M}$ e $\mathbf{M}^{*}$, vemos que 
a distância entre o átomo adsorvido e os átomos dos dímeros ( $\mathrm{I}$ e J) são muito semelhantes para as duas estruturas. A diferença está basicamente na distância do átomo de Ge ao átomo de Si da segunda camada (L), que no caso do sítio $\mathbf{M}$ é menor do que para o sítio $\mathbf{M}^{*}$. Esta diferença á a principal responsável pelo ordenamento energético destas duas estruturas. Fizemos um estudo da distribuição de carga total nestas estruturas, e observou-se que para o sítio $\mathbf{M}$, para o qual a distância entre o átomo de Ge e o átomo de Si da segunda camada é menor, existe uma densidade de carga entre os dois átomos. Apesar desta densidade não caracterizar uma ligação $\sigma$ como no caso das ligações do cristal, ela apresenta uma pequena quantidade de ligação para estes átomos. No caso do sítio $\mathbf{M}^{*}$, a distribuição total de carga possui uma densidade menor do que no caso do sítio $\mathbf{M}$. Tais distribuições podem ser vistas na Figura4.5. É esta diferença na densidade de carga que faz com que o sítio $\mathbf{M}$ seja mais estável do que o sítio $\mathbf{M}^{*}$.

Foram também estudadas as estruturas $\mathbf{M}$ e $\mathbf{M}^{*}$ sem o átomo de Germânio adsorvido, ou seja, estudamos as reconstruções das estruturas $\mathbf{M}$ e $\mathbf{M}^{*}$. A simulação foi feita com todos os átomos fixos. O que se observou foi que desta maneira a superfície $\mathbf{M}^{*}$ é mais estável do que a superfície $\mathbf{M}$, por uma diferença de $0.36 \mathrm{eV}$. Este resultado reforça a teoria de que o átomo adsorvido no sítio $\mathbf{M}$ é mais estável por causa de efeitos relativos ao átomo adsorvido e não relativos à superfície.

No caso do sítio $\mathbf{H}$, apesar de este não estar localizado exatamente no centro de dois dímeros da superfície, exite uma densidade de carga, aparentemente ligante, entre o átomo adsorvido e os quatro átomos de $\mathrm{Si}$ dos dois dímeros mais próximos. Estas ligações se assemelham muito com a ligação entre o átomo de Ge com o átomo de Si da segunda camada no caso do sítio M. Outro fator que indica que estas densidades de carga entre o átomo de Ge e os átomos de Si para o sítio $\mathbf{H}$ são ligações, é que não foi encontrado nenhum orbital flutuante sobre o átomo adsorvido.

Como foram encontradas duas posiçoes muito semelhantes ( $\mathbf{M}$ e $\mathbf{M}^{*}$ ), 


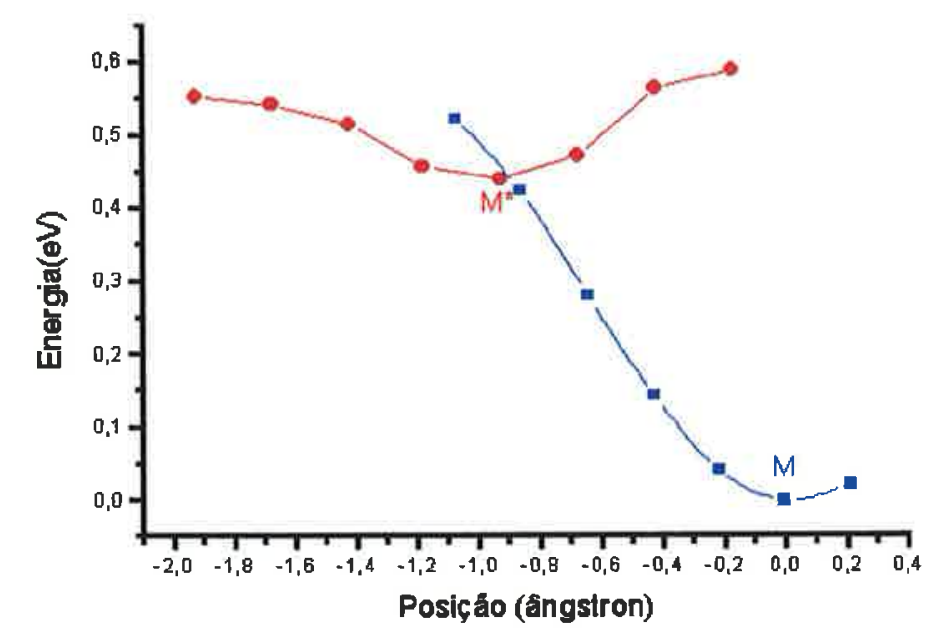

Figura 4.6: A curva em vermelho representa uma difusão com o deslocamento dos dímeros da superfície em fase, o que nos fornece o sítio metaestável $\mathbf{M}^{*}$. A curva azul representa uma difusão com o deslocamento fora de fase. O sítio mais estável $\mathrm{M}$ é tido como origem para a posição e para a energia. A posição se refere à difusão sobre uma reta que contém os sítios $\mathbf{M}$ e $\mathbf{M}^{*}$.

que podem ser interpretadas como sítios semelhantes, resolvemos encontrar a barreira para que houvesse difusão da posição $\mathbf{M}$ para a posição $\mathbf{M}^{*}$. Como já foi dito anteriormente, a distância entre estes dois sítios é de $0.86 \AA$. Foram tomadas três posições intermediárias para obter a barreira de difusão. $O$ resultado obtido foi muito interessante, visto que foram obrtidas duas curvas diferentes para esta difusão, uma partindo do sítio $\mathbf{M}$ indo para o sítio $\mathbf{M}^{*}$ e outra no sentido contrário. A diferença nestas duas curvas era somente o deslocamento dos dímeros de Silício da superfície.

Este resultado nos levou a inserir um novo grau de liberdade para a adsorção de átomos sobre superfícies. Além da posição $(\mathrm{x}, \mathrm{y})$, o deslocamento dos átomos da superfície também são muito importantes. As direfentes or- 
ganizações dos dímeros da superfície nos fornecem diferentes sítios ligantes (mínimos locais). Este resultado nos dá uma boa explicação de porquê Milman obteve um mínimo local como sendo o sítio mais estável.

As duas curvas para a difusão do átomo podem ser vistas na Figura4.6. Neste gráfico, observamos que os sítios $\mathbf{M}$ e $\mathbf{M}^{*}$ correspondem a mínimos para as configurações do deslocamento dos dímeros fora de fase e em fase, respectivamente. É possível que existam outras configurações metaestáveis, mas este não é o nosso objetivo. Os resultados de Brocks et al. mostram isto, visto que eles obtiveram um deslocamento para cima para a adsorção de átomos de Si sobre $\mathrm{Si}(100)$.

Especulamos que este novo grau de liberdade venha a ser importante na adsorção de átomos e molécluas sobre superfícies covalentes, como a superfície de $\mathrm{Ge}, \mathrm{SiGe}$, entre outras. Isto significa que muitos resultados reportados na. literatura devem ser revistos ou completados.

Para finalizar,supomos que o sítio metaestável $\mathbf{M}^{*}$ seja muito importante para a explicação de alguns fenômenos observados durante a formação de dímeros. Qin e Lagally observaram uma estrutura de dois átomos semelhante ao dímero $\mathbf{C}$, mas que não apresentava ligação entre os átomos de Ge. Especula-se que esta estrutura possa ser explicada considerando-se os graus de liberdade adicionais, relacionados ao deslocamento dos dímeros da superfície, para a formação de dímeros. Supomos também que o sítio $\mathbf{M}^{*}$ seja muito importante para o processo de crescimento de estruturas maiores.

\subsubsection{Dímeros}

Daremos aqui uma breve noção de alguns resultados recentes [33] para a adsorção de dímeros de Ge sobre $\mathrm{Si}(100)$.

Assim como no caso de dímeros de $\mathrm{Si}$ sobre $\mathrm{Si}(100)$, foram estudadas quatro estruturas distintas. Estas estruturas são as mesmas descritas na Figura 4.2. Uma imagem da estrutura relaxada de dímeros de Ge sobre

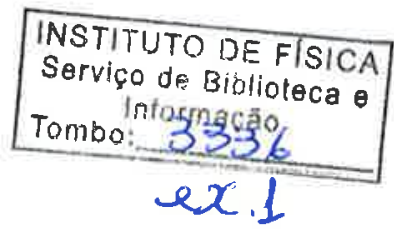


Tabela 4.2: Ângulos de torção de dímeros e distâncias entre átomos dos dímeros. As distâncias são dadas em ângstrons e os ângulos em graus.

\begin{tabular}{|c|c|c|c|c|c|c|c|c|}
\hline & \multicolumn{2}{|l|}{$\mathrm{B}$} & \multicolumn{2}{|l|}{ A } & \multicolumn{2}{|l|}{$\mathrm{C}$} & \multicolumn{2}{|l|}{$\mathrm{D}$} \\
\hline & dist. $(\AA)$ & Ângulo $\left(^{\circ}\right)$ & dist. $(\AA)$ & Ângulo $\left(^{\circ}\right)$ & dist. $(\AA)$ & Ângulo $\left({ }^{\circ}\right)$ & dist. $(\AA)$ & Ângulo( $\left.{ }^{\circ}\right)$ \\
\hline A & 2.32 & 19.2 & 2.31 & 19.2 & 2.28 & 17.0 & 2.28 & 16.9 \\
\hline $\mathrm{B}$ & 2.32 & 19.5 & 2.32 & 19.2 & 2.40 & 8.4 & 2.50 & 3.7 \\
\hline $\mathrm{C}$ & 2.32 & 19.4 & 2.32 & 19.1 & 2.39 & 5.8 & 2.50 & 3.0 \\
\hline $\mathrm{D}$ & 2.32 & 19.2 & 2.31 & 19.1 & 2.28 & 17.1 & 2.28 & 17.0 \\
\hline $\mathrm{E}$ & 2.32 & 20.4 & 2.32 & 18.1 & 2.28 & 17.3 & 2.28 & 17.0 \\
\hline $\mathrm{F}$ & 2.31 & 1.9 & 2.49 & 1.9 & 2.39 & 3.3 & 2.49 & 2.8 \\
\hline $\mathrm{G}$ & 2.32 & 2.2 & 2.39 & 1.5 & 2.40 & 5.5 & 2.50 & 3.7 \\
\hline $\mathrm{H}$ & 2.32 & 20.3 & 2.30 & 19.8 & 2.28 & 17.1 & 2.28 & 17.1 \\
\hline I & 2.47 & 0.2 & 2.46 & 5.7 & 2.69 & 0.1 & 2.39 & 0.3 \\
\hline $\mathrm{J}$ & 2.46 & - & 2.38 & - & 2.48 & - & 2.48 & - \\
\hline $\mathrm{L}$ & 2.50 & - & 2.40 & - & 2.47 & - & 2.48 & - \\
\hline $\mathrm{M}$ & 2.50 & - & 2.36 & - & 2.48 & - & 2.48 & - \\
\hline $\mathrm{N}$ & 2.46 & - & 2.44 & - & 2.48 & - & 2.48 & - \\
\hline
\end{tabular}

Si(100) pode ser vista na Figura4.7. As distâncias atômicas e os ângulos de deslocamento dos dímeros podem ser vistos na Tabela 4.2.

Ao contrário do caso de dímeros de $\mathrm{Si}$ sobre $\mathrm{Si}(100)$, o dímero mais estável encontrado foi o dímero $\mathbf{B}$, seguido pelo $\mathbf{A}, \mathbf{C}$ e $\mathbf{D}$. As diferenças de energias relativas ao dímero mais estável são $0.0,0.08,0.30$ e $1.00 \mathrm{eV}$. Estes valores foram obitdos através de cálculos utilizando quatro pontos especiais para a integração na Zona de Brillouin.

Observando a Figura4.7 e a Tabela 4.2, veremos que as distâncias entre os átomos dos dímeros da superfície são maiores do que as distâncias dos dímeros na superfície limpa quando um dímero de Ge é adsorvido entre duas colunas de dímeros. Já se o dímero é adsorvido sobre uma coluna de dímeros, a distância entre os átomos dos dímeros da superfície é similar aos valores da superfície limpa. Este é um dos fatores porque os dímeros adsorvidos sobre as colunas de dímeros são mais estáveis do que os adsorvidos entre as colunas de dímeros. Outro fator importante para tal ordenamento é que no caso dos 


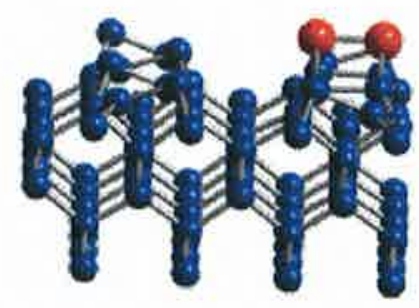

B
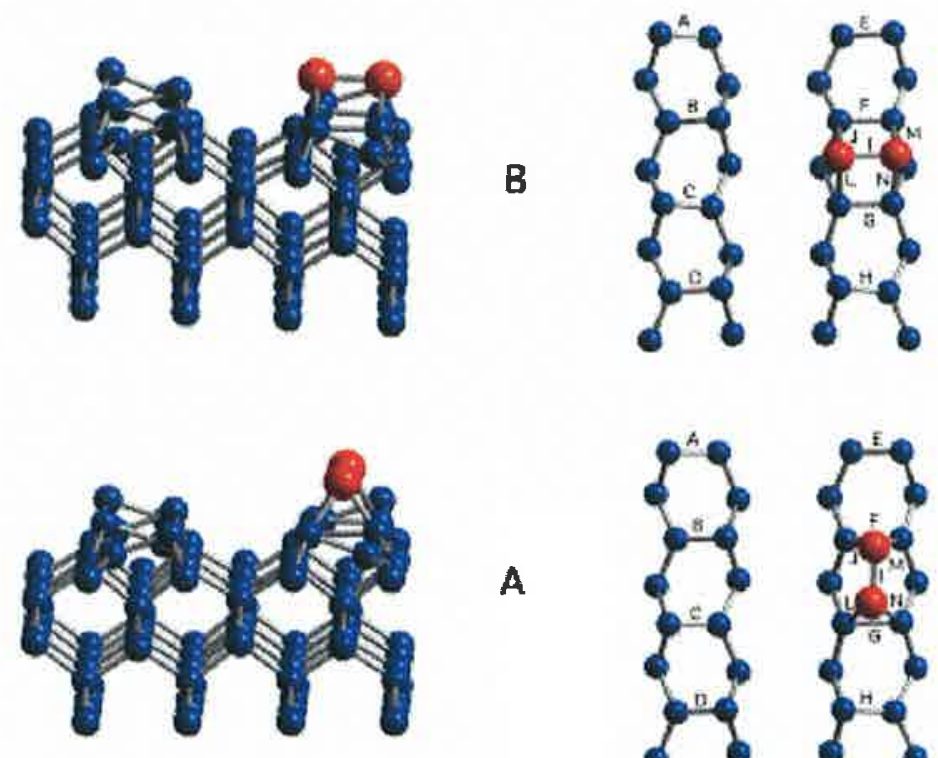

A
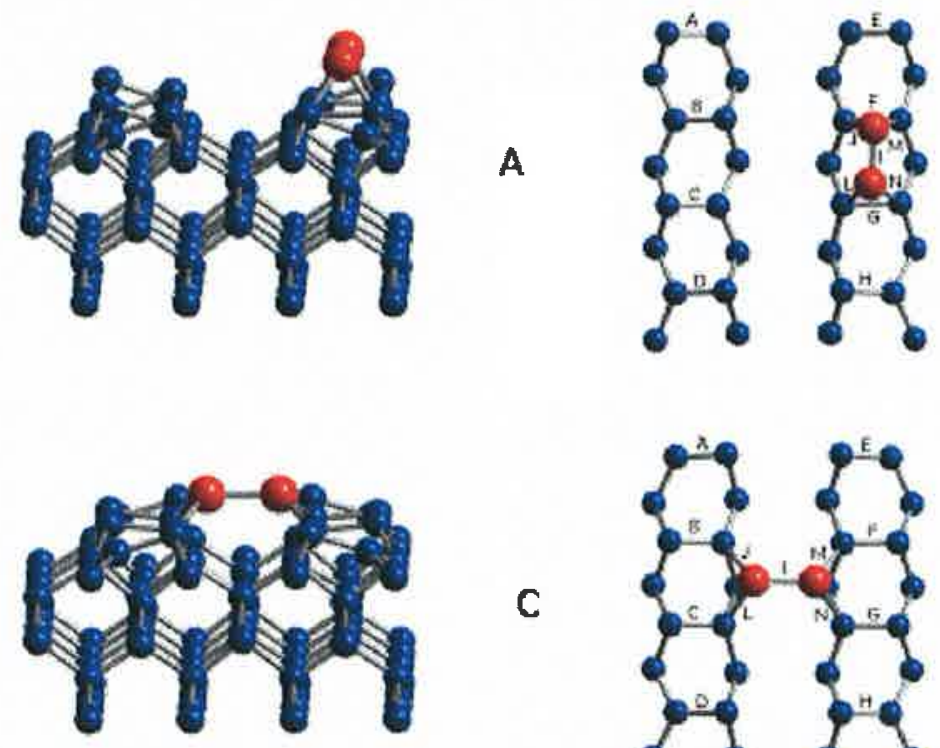

C
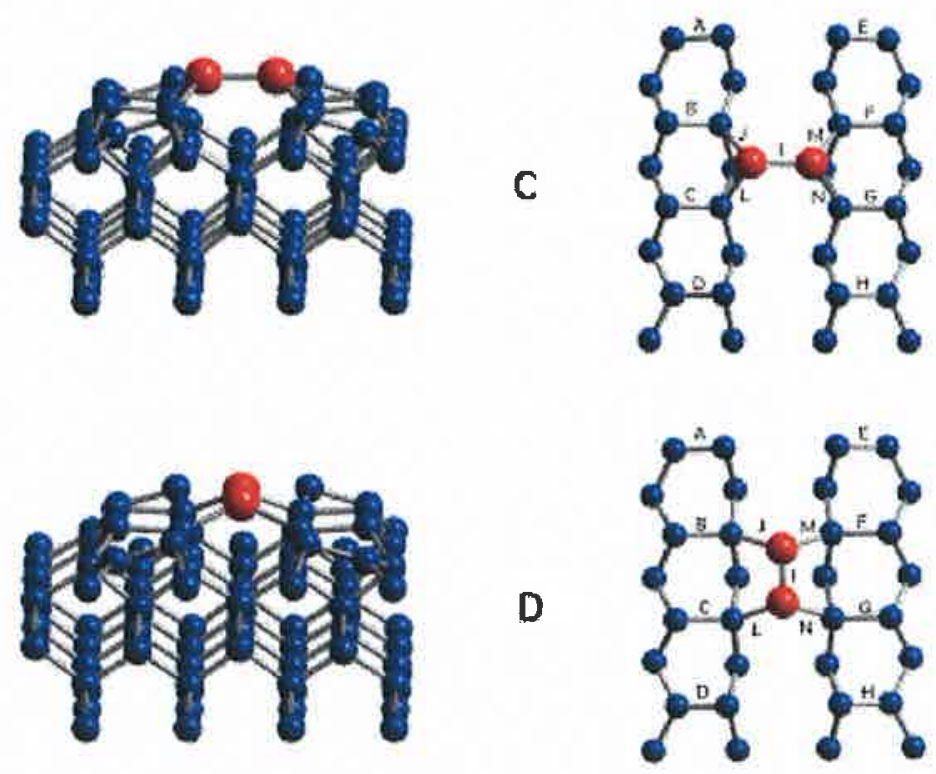

Figura 4.7: Imagens das quatro estruturas estudadas para a adsorção de dímeros de Ge sobre a superfície. As imagens da direita nos fornecem uma visão superior da estrutura. As letras servem de referência para as distâncias mostradas na Tabela4.2. 


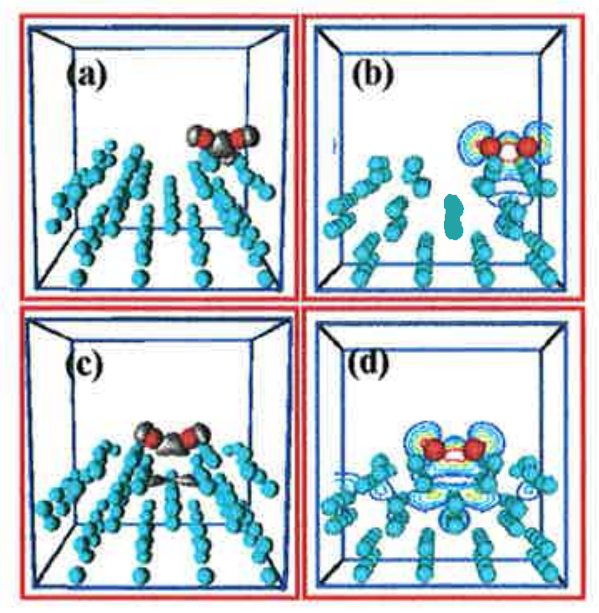

Figura 4.8: Distribuição de carga dos orbitais ligantes entre os átomos de Ge para os dímeros B e C. (a) e (b) distribuição de carga espacial e sobre um plano que passa pelos átomos de Ge, do orbital ligante entre os átomos de Ge, para o dímero B; (c) e (d) distribuição de carga espacial e sobre um plano que passa sobre os átomos de $\mathrm{Ge}$, para o orbital ligante entre os átomos de Ge do dímero $\mathrm{C}$.

dímeros sobre uma coluna de dímeros, a superfície fica com menos ligações flutuantes do que no caso dos dímeros entre duas colunas.

De acordo com os resultados de Qin e Lagally[25], o dímero $\mathbf{C}$ na verdade seria um par de átomos, como já foi mencionado anteriormente por nós. Para averiguar isto, foi feita uma análise dos orbitais ocupados para o dímero $\mathbf{C}$. Encontrou-se que a uma energia de $0.94 \mathrm{eV}$ abaixo do HOMO(Highest Occupied Molecular Orbital) havia uma distribuição de carga que caracterizava uma ligação entre os átomos deste dímero. A distribuição de carga deste orbital pode ser vista na Figura4.8. O que reforça o fato de que esta é uma ligação, é o fato de para o dímero $\mathbf{B}$ ter sido encontrada uma distribuição de carga a $0.50 \mathrm{eV}$ abaixo do HOMO com as mesmas características da distribuição de carga do dímero $\mathbf{C}$. Tal orbital pode ser visto na Figura4.8.

Foram feitas também algumas imagens teóricas de STM para que pudesse ser feita uma comparação mais precisa entre as estruturas obtidas por nós e 

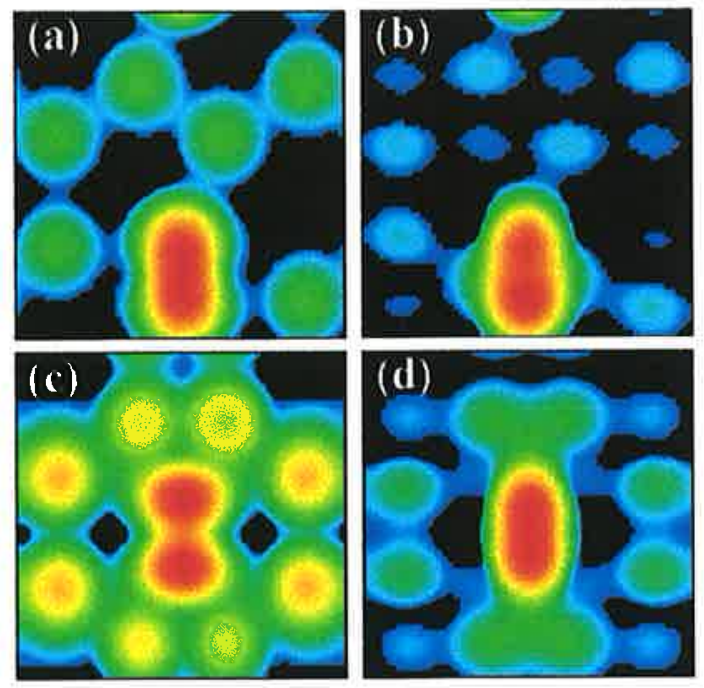

Figura 4.9: Imagens teóricas de STM dos orbitais cheios (a) e (c) e dos orbitais vazios (b) e (d); as letras (a) e (b) representam o dímero $\mathbf{B}$ e as letras (c) e (d) representam o dímero $\mathrm{C}$. $\mathrm{O}$ vermelho indica uma altura maior em relação ao azul.

as imagens obtidas por Qin e Lagally. As imagens foram geradas através da aproximação de Tersoff-Hamman, onde a corrente de tunelamento é proporcional à Densidade de Estados Local na posição onde a ponta de prova está, integrada sobre um "range" de energia da ordem da tensão aplicada à ponta de prova.

As imagens geradas podem ser vistas na Figura4.9. Estas imagens mostram a variação da altura da ponta à medida que esta vai varrendo a superfície, sendo que a corrente de tunelamento sempre é mantida constante. Como pode ser visto na figura, para o dímero $\mathbf{C}$, a imagem muda muito quando a tensão é invertida, sendo que na imagem dos orbitais ocupados (cheia), existe um sinal forte também sobre os átomos de $\mathrm{Si}$ ao redor do dímero do Ge, enquanto que na imagem dos orbitais desocupados (vazia) o sinal está mais localizado sobre o dímero de Ge. $\mathrm{O}$ dímero $\mathbf{B}$, entretanto, não mostra uma grande diferença entre imagens cheias e vazias. $\mathrm{Na}$ imagem cheia do dímero 
C, também se observa que o sinal sobre o dímero apresenta um decréscimo na intensidade entre os átomos do dímero. Este resultado concorda qualitativamente muito bem com os resultados de Qin e Lagally, que observam isto no seu "quase-dímero". Neste caso, uma concordância perfeita se torna quase impossível em virtude de para os resultados teóricos ser considerada a existência de uma ponta pontual.

\subsubsection{Trímeros}

Para três átomos de Germânio adsorvidos sobre a superfície, estudamos três configurações, que consideramos de extrema importância no processo de crescimento. Estas três estruturas podem ser observadas na Figura 4.10.

A configuração mais estável observada foi a T1, seguida pela T2 e T3. As diferenças de energia relativas à estrutura mais estável são $0.0,0.28,0.40 \mathrm{eV}$, respectivamente.

Os nossos resultados estão de acordo com resultados teóricos [22] e experimentais [28] para adsorção de Si sobre Si(100). Wingerden somente conseguiu observar a estrutura T1, através de STM e Yamasaki obteve que a estrutura $\mathbf{T} 1$ era realmente a mais estável, para Si sobre Si(100), entre várias estruturas estudadas.

A motivação para o estudo das estruturas T2 e T3 veio do trabalho de Wingerden, que aponta estas duas estruturas como sendo configurações precedentes à formação do trímero $\mathbf{T} 1$, como já foi descrito anteriormente.

As três estruturas são compostas por um dímero, que no caso dos trímeros T1 e $\mathbf{T 3}$ é o dímero $\mathbf{B}$ e no caso do trímero $\mathbf{T 2}$ é um dímero $\mathbf{A}$, mais um átomo adsorvido num sítio $\mathbf{M}$ próximo ao dímero. A posição do terceiro átomo adsorvido(monômero) concorda muito bem, em todos os casos, com o sítio $\mathbf{M}$ encontrado por nós. A diferença está somente na configuração dos dímeros da superfície, que para os trímeros T1 e T3 aparecem quase simétricos, em virtude do forte potencial imposto pelo dímero. Para o trímero 


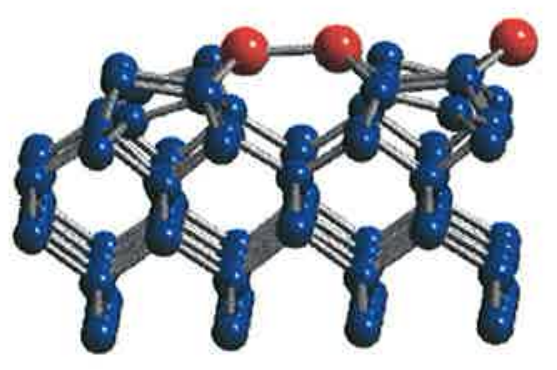

T1
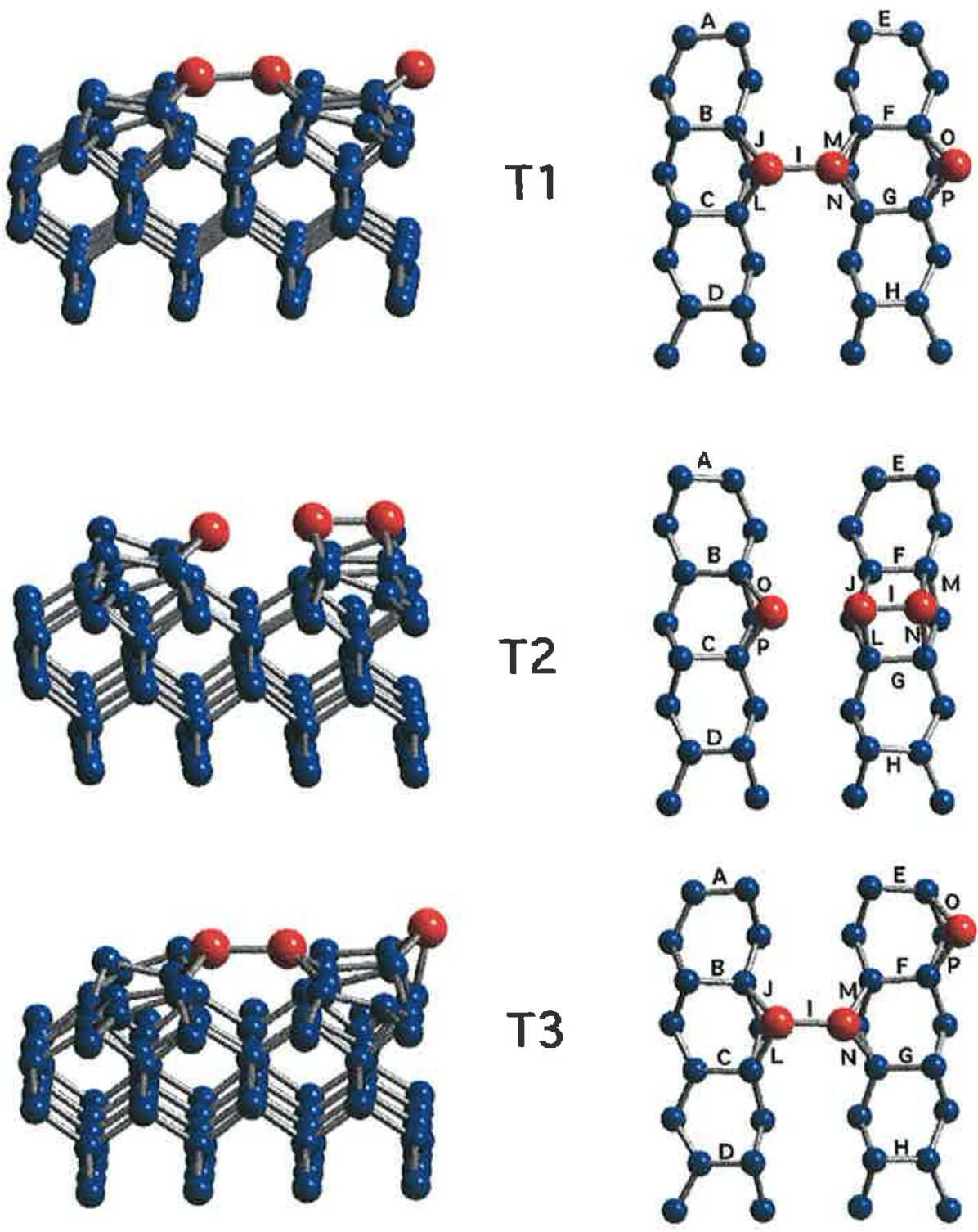

Figura 4.10: Imagens das três estruturas estudadas para a adsorção de trímeros de Ge sobre a superfície. As imagens da direita nos fornecem uma visão superior das estruturas. As letras servem de referência para as distâncias mostradas na Tabela4.3. 
Tabela 4.3: Ângulos de torção de dímeros da superfície e distâncias entre átomos para trímeros adsorvidos. As letras estão indicadas na Figura4.10. As distâncias são dadas em ângstrons e os ângulos em graus.

\begin{tabular}{|c|c|c|c|c|c|c|}
\hline & \multicolumn{2}{|c|}{ T1 } & \multicolumn{2}{|c|}{ T2 } & \multicolumn{2}{c|}{ T3 } \\
\cline { 2 - 7 } & dist. $(\AA)$ & Ángulo $\left(^{\circ}\right)$ & dist. $(\AA)$ & Ângulo $\left(^{\circ}\right)$ & dist. $(\AA)$ & Angulo $\left({ }^{\circ}\right)$ \\
\hline A & 2.28 & 16.7 & 2.31 & 19.2 & 2.28 & 17.3 \\
\hline B & 2.42 & 9.2 & 2.32 & 14.0 & 2.43 & 13.3 \\
\hline C & 2.41 & 8.8 & 2.39 & 11.8 & 2.35 & 7.1 \\
\hline D & 2.28 & 16.7 & 2.30 & 18.9 & 2.28 & 17.7 \\
\hline E & 2.27 & 16.1 & 2.32 & 20.0 & 2.33 & 0.8 \\
\hline F & 2.39 & 5.0 & 2.31 & 0.8 & 2.40 & 4.0 \\
\hline G & 2.39 & 5.0 & 2.31 & 3.0 & 2.42 & 7.0 \\
\hline H & 2.27 & 15.9 & 2.32 & 20.1 & 2.27 & 14.5 \\
\hline I & 2.63 & 1.4 & 2.51 & 1.0 & 2.69 & 0.9 \\
\hline J & 2.47 & - & 2.46 & - & 2.50 & - \\
\hline L & 2.47 & - & 2.51 & - & 2.46 & - \\
\hline M & 2.46 & - & 2.49 & - & 2.45 & - \\
\hline N & 2.46 & - & 2.46 & - & 2.48 & - \\
\hline O & 2.43 & - & 2.43 & - & 2.43 & - \\
\hline P & 2.43 & - & 2.48 & - & 2.44 & - \\
\hline
\end{tabular}


T2, esta interação é pequena, o que faz com que os dímeros da superfície fiquem numa configuração similar à que ocupam quando um monômero é adsorvido no sítio $\mathbf{M}$.

Além do estudo estrutural da superfície, fizemos também um estudo da distribuição de carga nestas estruturas e de imagens de STM delas.

Através da análise da estrutura eletrônica, observamos que não existe nenhuma tendência de ligação entre o dímero adsorvido e o terceito átomo, em nenhuma das configurações estudadas. Portanto, este não é um fator relevante para o ordenamento energético das estruturas. Um fator importante para tal ordenamento é o número de ligações flutuantes dos átomos de $\mathrm{Si}$ da superfície. No caso dos trímeros $\mathbf{T} 1$ e $\mathbf{T} 2$, existem duas ligações flutuantes nos átomos de Si. Estas estão no átomo superior dos dímeros $\mathrm{B}$ e $\mathrm{C}$ da superfície. O trímero T3 possui quatro ligações flutuantes, nos átomos superiores dos dímeros ${ }^{2}$ B, C, E e G da superfície. Isto, entretanto, explica somente porque o trímero T3 é menos estável, mas não porque o trímero T1 é mais estável do que o trímero T2. Este ordenamento deve-se provavelmente à intensidade das ligações dos átomos de Ge do dímero $\mathbf{C}$ no trímero $\mathbf{T 1}$, que deve ser maior do que a intensidade das ligações dos átomos de Ge do dímero $\mathbf{B}$ no caso do trímero $\mathbf{T} \mathbf{2}$.

Este ordenamento dos trímeros é muito interessante, visto que o trímero mais estável é formado por um dímero $\mathbf{C}$ mais um monômero $\mathbf{M}$, enquanto que as estruturas de dímeros e monômeros isolados mais estáveis são, respectivamente, $\mathbf{B}$ e $\mathbf{M}$. Portanto, o ordenamento dos trímeros não exibe uma relação direta com o ordenamento energético de dímeros e monômeros.

Na Figura4.11, pode-se observar a distribuição de carga de alguns orbitais, responsáveis pelas ligações dos átomos de Germânio. Nota-se, através desta figura, que as distribuições de carga sobre o dímero e sobre o monômero pouco mudam em relação ao dímero e o monômero isolados.

Um fato interessante que é observado experimentalmente é que, quando

\footnotetext{
${ }^{2}$ Observar Figura 4.10.
} 


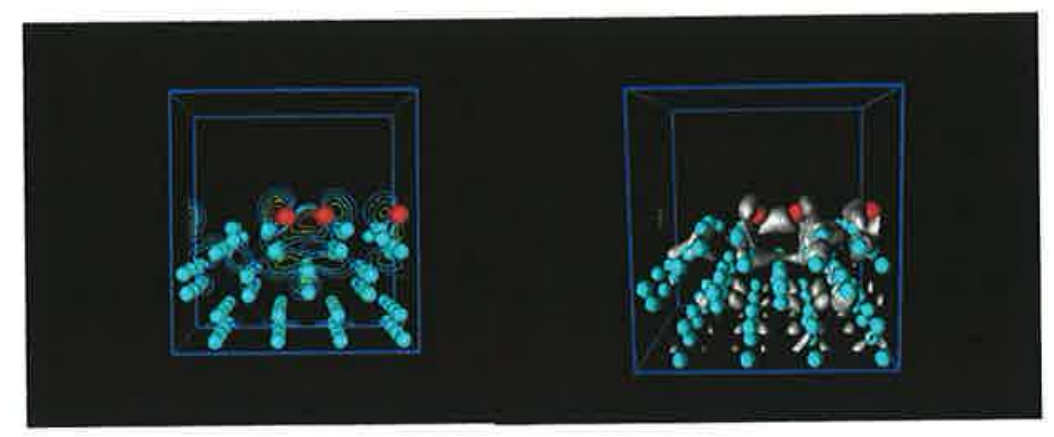

Figura 4.11: Densidade de carga referente aos orbitais relevantes para as ligações dos átomos de Germânio. A figura da esquerda mostra a distribuição de carga num plano que passa pelo átomo de Ge e a da direita mostra a distribuição de carga espacial pela estrutura. Ambas se referem ao trímero T1.
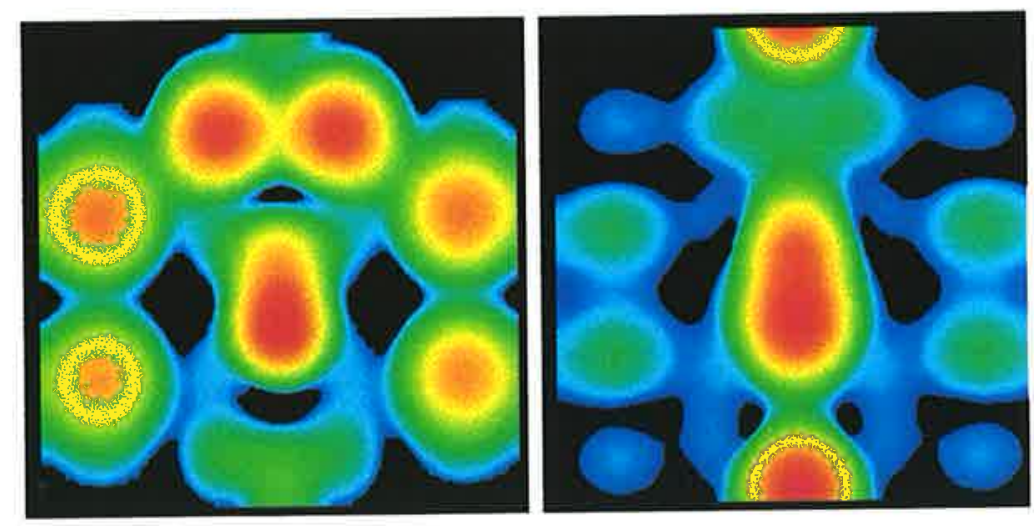

Figura 4.12: Imagens de STM teóricas da estrutura T1. A imagem da esquerda representa os orbitais cheios e da direita os vazios. O terceiro átomo adsorvido é invisível nas imagens cheias, o que está de acordo com resultados experimentais. 
um átomo está adsorvido próximo a uma ilha maior, este não aparece em imagens cheias de STM, e sim somente nas imagens vazias. Nós resolvemos tentar observar tal fato, utilizando o trímero $\mathrm{T} 1$, onde o terceiro átomo faria o papel do átomo invisível. Como pode ser visto na Figura4.12, encontramos uma imagem muito parecida com os resultados experimentais. Observando a imagem cheia, pode-se observar um sinal forte no centro, referente ao dímero de Ge. Ao redor dele, existem vários sinais, que se referem aos átomos de Silício da superfície. Entratanto, nenhum sinal pode ser visto na parte inferior da figura, que é a posição onde o terceiro átomo está adsorvido. Portanto, este é o átomo "invisível". Já na imagem cheia, observa-se que o sinal está somente sobre os átomos de Germânio, numa mesma intensidade para o dímero e para o monômero. 


\section{Capítulo 5}

\section{Conclusão}

Durante os estudos da adsorção de um átomo de Germânio sobre a superfície (100) do Silício, observamos que a quantidade de mínimos locais para esta adsorção era muito grande.

Obtivemos alguns destes mínimos locais, sendo que o sítio mais estável encontrado foi o sítio $\mathbf{M}$, seguido do sítio $\mathbf{M}^{*}$ e do sítio $\mathbf{H}$. $\mathbf{M}^{*}$ é um sítio metaestável que havia sido descrito como sendo um mínimo global em outros trabalhos. Isto ocorreu em virtude da não inclusão dos graus de liberdade da superfície, introduzidos por nós e relativos ao deslocamento dos dímeros de Silício da superfície.

Este novo grau de liberdade introduz uma série de mínimos locais sobre a superfície, que devem ser examinados para se obter uma conclusão definitiva sobre as posições mais estáveis para a adsorção de átomos.

Foram feitos também alguns estudos sobre dímeros e trímeros de Ge sobre a superfície. Observou-se que o trímero mais estável não é formado pela junção do dímero e monômero mais estáveis. A estrutura mais estável é constituída por um dímero $\mathbf{C}$ e um monômero $\mathbf{M}$. Foram feitas imagens teóricas de STM, que concordam muito bem com resultados experimentais.

Os resultados apresentados neste trabalho, são importantes em virtude de apresentarem um novo ítem que deve ser observado quando se trabalha 
em adsorção de átomos sobre superfícies, em particular Ge sobre a superfície (100) do Silício, A observação do deslocamento dos dímeros da superfície pode nos trazer novas informações sobre barreiras de difusão e sobre sítios ligantes. Seria muito interessante, num estudo futuro, a observação destes efeitos em outras superfícies, inclusive superfícies não covalentes (como GaAs, ...), e para outros tipos átomos sendo adsorvidos, a fim de comprovar se estes resultados podem ser transferidos para outros sistemas. Supomos inclusive que estes graus de liberdade sejam importantes na adsorção de pequenas moléculas sobre superfícies.

O trabalho realizado por nós seguiu o que há de mais moderno no que se refere a cálculos quânticos de sólidos, sendo que refletiram muito bem propriedades básicas bem definidas das estruturas estudadas. Isto nos permitiu uma comparação muito boa com resultados experimentais, o que reafirma a qualidade do nosso trabalho. 


\section{Bibliografia}

[1] Bockstedte M., Kley A., Neugebauer J. e Scheffler M. Density-Functional theory calculations for poly-atomic systems: electronic structure, stat$i c$ and elastic properties and $a b$ initio molecular dynamics. Computer Physics Communications 107, 187-222(1997).

[2] Hohenberg P. e Kohn W. Inhomogeneous Electron Gas. Physical Review 136, B864-B871(1964).

[3] Kohn W. e Sham L. J. Self Consistent Equations Including Exchange and Correlation Effects. Physical Review 140, A1133-A1138(1965).

[4] Hamann D. R., Schlüter M. e Chiang C. Norm-Conserving Pseudopotentials. Physical Review Letters 43, 1494-1497(1979).

[5] Bachelet G. B., Hamann D. R. e Schlüter M. Pseudopotentials that work: From $H$ to $P$ u. Physical Review B 26, 4199-4228(1982).

[6] Troullier N. e Martins J. L. Efficient pseudopotentials for plane-wave calculations. Physical Review B 43, 1993-2006(1991).

[7] Kleinman L. e Bylander D. M. Efficacious Form for Model Pseudopotentials. Physical Review Letters 48, 1425-1428(1982).

[8] Ihm J. , Zunger A. e Cohen M. Momentum-space formalism for the total energy of solids. Journal of Physics C 12, 4409-4422(1979). 
[9] Baldereschi A. Mean-Value Point in the Brillouin Zone. Physical Review B 7, 5212-5215(1973).

[10] Feynman R. P., Forces in Molecules. Physical Review. 56, 340 (1939).

[11] Car R. e Parrinello M. Unified Approach for Molecular Dynamics and Density-Functional Theory. Physical Review Letters 55, 24712474(1985).

[12] Payne M. C., Joannopoulos J. D., Allan D. C., Teter M. P. e Vanderbilt D. H. Molecular Dynamics and Ab Initio Total Energy Calculations. Physical Review Letters 56, 2656(1986).

[13] Dabrowski J. e Scheffler M. Self-Consistent Study of the Electronic and Structural Properties of the Clean Si(001)(2x1) Surface. Applied Surface Science 56-58, 15-19(1992).

[14] Ramstad A., Brocks G. e Kelly P. J. Theoretical Study of the Si(100) Surface Reconstruction. Physical Review B 51, 14504-14523(1995).

[15] Fritsch J. e Pavone P. Ab Initio Calculation of the Structure, Electronic States, and the Phonon Dispersion of the Si(100) Surface. Surface Science 344, 159-173(1995).

[16] Kubby J. A. e Boland J. J. Scanning Tunneling Microscopy of Semiconductor Surfaces. Surface Science Reports 26, 61-204(1996).

[17] Boland J. J. e Weaver J. H., A Surface View of Etching. Physics Today, 34-40(aug/1998).

[18] Bechstedt F. e Enderlein R. Semiconductor Surfaces and Interfaces. Akademie-Verlag Berlin, (1988).

[19] Brocks G., Kelly P. J. e Car R. Binding and Diffusion of a Si Adatom on the Si(100) Surface. Physical Review Letters 66, 1729-1732(1991). 
[20] Srivastava D. e Garrison B. J., Adsorption and diffusion dynamics of a Ge adatom on the Si(100)(2x1) surface. Physical Review B 46, 1472$1479(1992)$.

[21] Smith A. P., Wiggs J. K., Jónsson H., Yan H., Corrales L. R., Nachtigall P. e Jordan K. D. Si adatom binding and diffusion on the Si(100) surface: Comparison of ab initio, semiempirical and empirical potential results. Journal Chemical Physics 102, 1044-1056(1995).

[22] Yamasaki T., Uda T. e Terakura K. Initial Process of Si Homoepitaxial Growth on Si(001). Physical Review Letters 76, 2949-2952(1996).

[23] Milman V., Jesson D. E., Pennycook S. J., Payne M. C., Lee M. H. e Stich I. Large scale ab initio study of the binding and diffusion of a Ge adatom on the Si(100) surface. Physical Review B50, 2663-2666(1994).

[24] Brocks G. e Kelly P. J. Dynamics and Nucleation of Si Ad-dimers on the Si(100) Surface. Physical Review Letters 76, 2362-2365(1996).

[25] Qin X. R. e Lagally M. G. Adatom Pairing Structures for Ge on Si(100): The Initial Stage of Island Formation. Science 278, 1444-1447(1997).

[26] Borovsky B., Krueger M. e Ganz E. Piecewise diffusion of the silicon dimer. Physical Review B 59, 1598-1601(1999).

[27] Lee G.-D., Wang C. Z., Lu Z. Y. e Ho K. M. Ad-Dimer Diffusion between Trough and Dimer Row on Si(100). Physical Review Letters 81, 5872$5875(1998)$.

[28] Wingerden J. van, Dam A. van, Haye M. J., Scholte P. M. L. O. e Tuinstra F. Room-temperature growth of submonolayers of silicon on Si(100) studied with scanning tunneling microscopy. Physical Review B 55, 4723-4730(1997). 
[29] Borovsky B., Krueger M. e Ganz E. Diffusion of the Silicon Dimer on Si(001): New Possibilities at 450K. Physical Review Letters 78, 42294232(1997).

[30] Swartzentruber B. S. Kinetics of Si monomer trapping at steps and islands on Si(001). Physical Review B 55, 1322-1325(1997).

[31] Mo Y.-M. and Lagally M. G. Anisotropy in surface migrations of Si and Ge on Si(001). Surface Science 248, 313-320(1991).

[32] Dalpian G. M., Janotti A., Fazzio A. e da Silva A. J. R., Initial Stages of Ge Growth on Si(100): Ad-Atoms, Ad-Dimers, and Ad-Trimers. Physica B 273, 589-592 (1999).

[33] Janotti A. Tese de Doutoramento apresentada ao Instituto de Física da USP (1999). 